\title{
Validación de modelos constitutivos avanzados de comportamiento mecánico para la arcilla estructurada de Brasilia
}

\author{
Validation of advanced constitutive models for the mechanical behaviour of Brasilia's \\ structured clay
}

Fecha de entrega: 13 de enero 2014

Fecha de aceptación: 28 de mayo 2014

\section{Cristhian Mendoza ${ }^{1}$, Márcio Farias ${ }^{2}$ y Renato P. da Cunha ${ }^{2}$}

\begin{abstract}
${ }^{1}$ Facultad de Ingeniería, Programa de Ingeniería Civil, Universidad Piloto de Colombia, Carrera $9 \mathrm{~N}^{\circ} 45 \mathrm{~A}-44$, Edificio académico, tercer piso, Bogotá, Colombia, cristhian-mendoza@unipiloto.edu.co

${ }^{2}$ Departamento de Ingeniería Civil y Ambiental, Facultad de Tecnología, Universidad de Brasilia, Campus Universitario Darcy Ribeiro, Brasília - CEP 70910-900 - Prédio SG-12, Brasil, muniz@unb.br, rpcunha@unb.br.
\end{abstract}

La mayoría de los modelos constitutivos inicialmente no tomaron en cuenta el efecto de la estructura y la cementación del suelo. Sin embargo, en las últimas décadas estas variables han sido incorporadas en varios modelos constitutivos para describir el comportamiento mecánico del suelo en estado natural. Para incluir estas variables es necesario desarrollar leyes de estructura que reproduzcan adecuadamente el comportamiento del material. En este artículo se analiza el comportamiento de la arcilla estructurada y cementada de la ciudad de Brasilia por medio de ensayos triaxiales y simulaciones numéricas. Para realizar las simulaciones se escogieron los modelos Cam Clay con estructura, Subloading Cam Clay e Hipoplasticidad con estructura combinados con dos leyes de estructura. Los dos primeros están basados en elastoplasticidad y el último en hypoplasticidad. De las simulaciones, se obtuvieron varias virtudes y defectos en comparación con los ensayos realizados. Finalmente, se discute ampliamente cuál es el modelo más conveniente para simular el comportamiento mecánico de la arcilla de la ciudad de Brasilia.

Palabras claves: ensayo triaxial, modelo elastoplástico, modelo hipoplástico, arcilla estructurada de Brasilia.
Themajorityoftheclassicalconstitutivemodelsforsoilsdoes not take into account the influence of the soil's structure and cementation. However, in the last decades these variables have been considered in various constitutive models to describe the mechanical behaviour of undisturbed soils. To include these variables it is necessary the development of structure laws able to model appropriately the material behaviour. In this paper, the behaviour of a structured and cemented clay from the city of Brasilia is analysed by means of triaxial tests and numerical simulations. To perform numerical simulations the models Cam Clay with structure, Subloading Cam Clay and Hypoplasticity with structure all of them combined with two structure laws, were chosen. The first two are based on elastoplasticity while the last one is based on hypoplasticity. As a result of the simulation process, advantages and drawbacks of each constitutive model were observed when compared with the test results. Finally, a discussion is presented in order to define the most appropriate model to simulate the mechanical behaviour of Brasilia's clay.

Keywords: triaxial tests, elastoplastic model, hypoplastic model, Brasilia's structured clays.

et al., 2005). Esto da como resultado que una gran proporción de los suelos encontrados en nuestro planeta tienen estas características (Melfi, 1997). En este artículo se estudia el suelo de la ciudad de Brasilia, el cual posee 
algunas características especiales. Es un depósito de suelo tropical arcilloso residual altamente intemperizado (laterítico), con una fuerte presencia de hierro y aluminio debido a procesos de lixiviación en las capas superiores. Esto da como resultado la creación de uniones entre partículas formando grumos (Figura 1a). La unión de estos grumos genera una estructura cementada con granos del tamaño de una arena (Figura 1b). En este material se han reportado relaciones de vacíos máximas alrededor de 2.0 y permeabilidades de $10^{-3}$ a $10^{-4} \mathrm{~m} / \mathrm{s}$ (Camapum et al., 2006). La estructura es altamente inestable bajo incrementos en los esfuerzos externos o variaciones en el contenido de humedad, lo que genera fuertes cambios de volumen conocido técnicamente como fenómeno de colapso. Adicionalmente, se han encontrado cambios significativos en el comportamiento mecánico del suelo en estado natural o reconstituido (Camapum et al., 2006).

a)

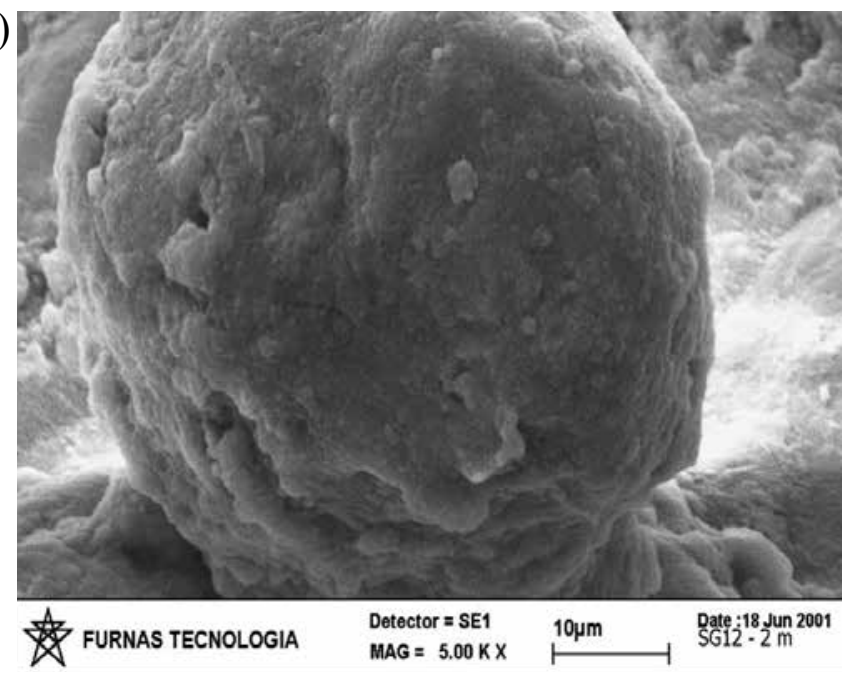

b)

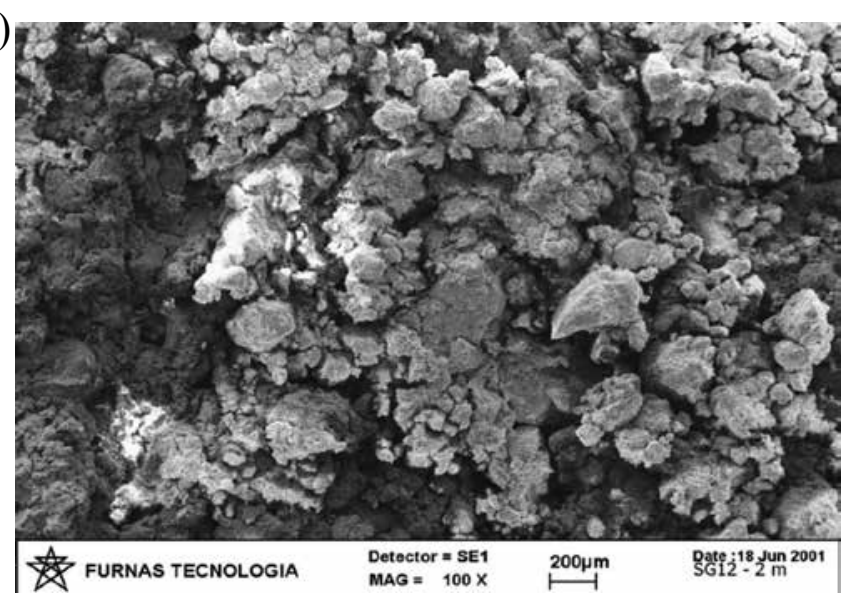

Figura 1. a) Estructura de suelo laterítico y b) grumo de partículas cementadas (Camapum et al., 2006 y Guimarães, 2002)
Las anteriores características crean la necesidad de comprender el comportamiento mecánico del suelo de Brasilia y entender cuál modelo reproduce mejor las características de este suelo cementado y estructurado. Para abordar este problema, inicialmente se realizaron ensayos de laboratorio bajo condiciones drenadas y no drenadas en muestras de arcilla obtenida en estado natural. A continuación se estudiaron e implementaron modelos constitutivos que tienen en cuenta la estructura y la cementación (Cam Clay con estructura, Hipoplasticidad con estructura y Subloading Cam Clay). Posteriormente se hizo la simulación de los ensayos realizados en este trabajo, lo cual permite evaluar cuál de los modelos implementados reproduce mejor el comportamiento de este tipo de suelo.

El artículo se divide en cinco secciones. La primera corresponde a los ensayos de laboratorio para determinar las características mecánicas del suelo estudiado. La segunda presenta los modelos constitutivos estudiados en este trabajo, su funcionamiento, implementación y comprobación. La tercera presenta las simulaciones de los ensayos realizados en este trabajo con cada uno de los tres modelos implementados. La cuarta sección presenta los resultados con su respectivo análisis e implicaciones prácticas y finalmente, se resaltan los elementos más importantes del trabajo desarrollado.

\section{Ensayos de laboratorio}

Para el estudio del comportamiento mecánico de la arcilla de Brasilia fueron obtenidas dos muestras en bloques de 30x30x30 cm, a $3 \mathrm{~m}$ de profundidad, localizadas en Guará (perteneciente al Distrito Federal, región centro oeste de Brasil) a las afueras de Brasilia. Las coordenadas aproximadas son $15^{\circ} 48^{\prime} 59^{\prime \prime} \mathrm{S}$ y $47^{\circ} 57^{\prime} 58^{\prime \prime} \mathrm{O}$ y una elevación de $1084 \mathrm{msnm}$. Una de las características de este sitio es la presencia de arcillas porosas, estructuradas y cementadas de la ciudad de Brasilia (Mendoza, 2013). Con estas muestras se realizaron ensayos de caracterización del material y ensayos de comportamiento mecánico, específicamente ensayos triaxiales. Con los resultados de estos ensayos se clasificó el suelo y se determinaron sus propiedades mecánicas. Esta información se utilizó para simular el comportamiento del suelo con los modelos constitutivos estudiados.

Los ensayos de caracterización sobre los bloques de suelo 
dieron los siguientes resultados: humedad natural con un valor promedio de $32.4 \%$, límite líquido de $42 \%$, límite de plasticidad de $30 \%$ (índice de plasticidad de $12 \%$ ), peso unitario $14.85 \mathrm{kN} / \mathrm{m}^{3}$, peso unitario seco de $11.39 \mathrm{kN} / \mathrm{m}^{3}$, peso específico de los sólidos de $26.69 \mathrm{kN} / \mathrm{m}^{3}$. Se realizó una granulometría por el método de sedimentación con los siguientes porcentajes: arena $16.4 \%$, limo $11.3 \%$ y arcilla $72.3 \%$. De lo anterior, se clasifica el suelo como arcilla.

Para la caracterización geomecánica del suelo se realizaron diez ensayos triaxiales en condiciones saturadas. En algunos ensayos fueron realizados cambios de velocidad de deformación, relajamiento y cambios en las trayectorias de esfuerzos. Esto ayudó a obtener los parámetros de los modelos constitutivos usados para la simulación de este tipo de suelo. Con los resultados de los ensayos se realizó una calibración de los modelos constitutivos y se evaluó el desempeño de éstos.

Se realizaron tres ensayos de consolidación anisotrópicos CA variando las trayectorias de esfuerzos $\eta=q / p^{\prime}=0.3,0.5$ y 0.0-0.5 en muestras naturales y un ensayo en una muestra reconstituida con una relación $\eta=q / p^{\prime}=0.3$. En la Figura 2 se muestra las trayectorias de esfuerzos realizadas y los esfuerzo de preconsolidación calculados por el método de Casagrande.

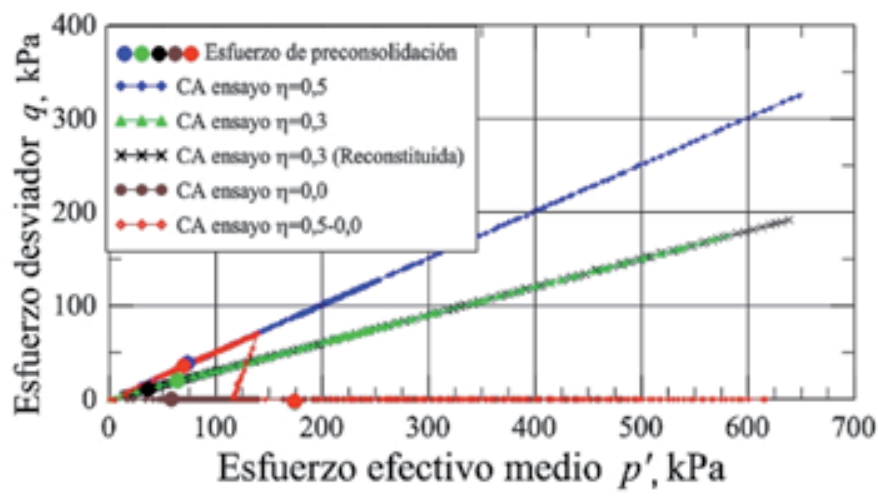

Figura 2: Trayectorias de esfuerzos en los ensayos de consolidación en triaxial y esfuerzos de preconsolidación calculados

El primer ensayo fue una consolidación triaxial con una relación de esfuerzos de $\eta=0.3$ y una relajación (cambio de esfuerzo efectivo sin cambio de volumen) durante el ensayo. Este ensayo está presentado en las Figuras 2 y 3. La trayectoria de esfuerzos y el punto del esfuerzo de preconsolidación se muestran en la Figura 2. Además, se realizó un ensayo en una muestra reconstituida con la misma relación de esfuerzos $(\eta=0.3)$, como se presenta en las Figuras 2 y 3 . En la Figura 3a se observa el efecto de la estructura en el suelo como la diferencia entre la línea de consolidación de la muestra reconstituida y la línea de consolidación de la muestra natural. El mismo efecto de estructura se observa en la Figura 4a al ver la diferencia entre la línea de consolidación del ensayo y la línea punteada obtenida del ensayo en la muestra reconstituida. Como resultado de lo anterior se observa un cambio del esfuerzo de preconsolidación causado por la estructura del suelo de $44 \mathrm{kPa}$ (intersección de la línea de consolidación normal sin estructura con la línea de consolidación del ensayo con estructura) a $70 \mathrm{kPa}$ (esfuerzo de preconsolidación calculado por un método clásico). En la Figura 3 b se ilustra la evolución del esfuerzo efectivo medio con el tiempo de relajación. Se percibe un decaimiento exponencial (línea roja) del esfuerzo efectivo en el tiempo hasta un punto donde la curva se vuelve asintótica. Después de la relajación se prosigue con la consolidación hasta un esfuerzo efectivo medio de $p^{\prime}=535 \mathrm{kPa}$, a partir de este esfuerzo se realiza una descarga hasta $5 \mathrm{kPa}$ y se vuelve a realizar un recarga hasta $p^{\prime}=580 \mathrm{kPa}$ (Figura 3a).

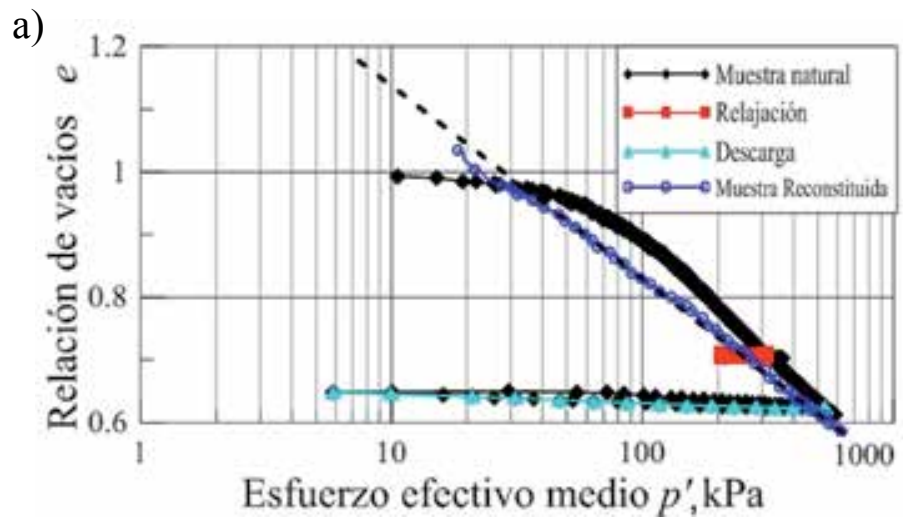

b)

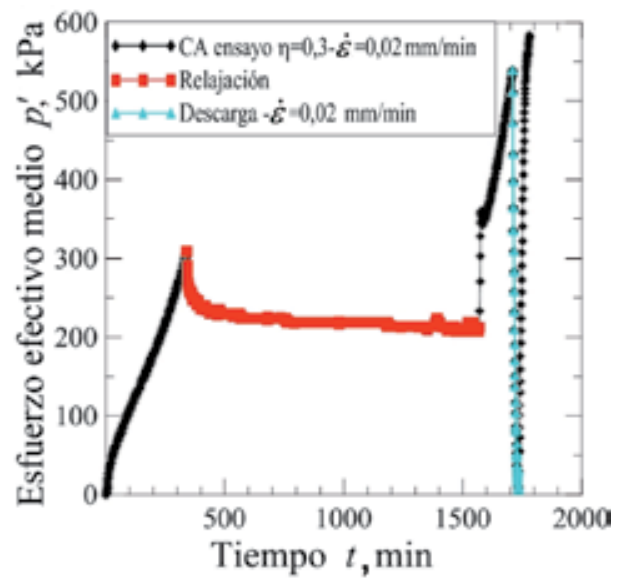

Figura 3: Ensayos de consolidación triaxial con $\eta=0.3$, a) curvas de compresibilidad y b) esfuerzo efectivo medio versus tiempo 
Posteriormente se realizó un ensayo con una relación de esfuerzos $\eta=q / p^{\prime}=0.5$ y cambios de velocidad de deformación durante el ensayo. Los cambios de velocidad se pueden apreciar en el plano de esfuerzo efectivo medio y relación de vacíos (Figura 4a), iniciando el ensayo a una velocidad de $\dot{\varepsilon}=0.01 \mathrm{~mm} / \mathrm{min}$, y posterior cambio a $\dot{\varepsilon}=0.001 \mathrm{~mm} / \mathrm{min}$ con el fin de obtener una relación de velocidades igual a 10 . De este ensayo se observa un cambio en las líneas de consolidación con el cambio de velocidad. Lo anterior ilustra el concepto propuesto por Tatsuoka et al. (2000) que llama "isotacas" a las líneas de consolidación sometidas a la misma velocidad. Los resultados concuerdan con el trabajo presentado por Nakai et al. (2009) en el cual tiene diferentes líneas de consolidación con el cambio de velocidad, pero estas líneas no son paralelas en la parte influenciada por la estructura. Por otra parte, en la Figura $4 \mathrm{~b}$ se grafica el cambio de volumen de la muestra en el tiempo y se observa que la salida de agua es lineal en el tiempo y las variaciones en la inclinación dependen de la velocidad. Con esto se puede decir que el cambio de volumen es función de la velocidad para pequeñas deformaciones. La trayectoria de esfuerzos y el punto de preconsolidación están en la Figura 2. En la Figura 4a se puede apreciar el cambio del esfuerzo de preconsolidación debido al efecto de la estructura al igual que en el ensayo anterior variando de 50 a $75 \mathrm{kPa}$.

En el último ensayo se realizó una consolidación con una relación de esfuerzos $\eta=0.5$ hasta un esfuerzo efectivo medio igual a $p^{\prime}=140 \mathrm{kPa}$ y una velocidad de deformación constante $\dot{\varepsilon}=0.02 \mathrm{~mm} / \mathrm{min}$. Después se realizó una descarga hasta alcanzar una condición isotrópica y se continuó con la consolidación con una relación de esfuerzos isotrópica $\eta=$ 0.0 , hasta un esfuerzo efectivo medio de $p^{\prime}=590 \mathrm{kPa}$, con la misma velocidad de deformación (Figura 2). En la Figura 5a se muestra el cambio del esfuerzo de preconsolidación con el cambio de trayectoria de esfuerzos. En la Figura 5b se presenta la curva de esfuerzo efectivo medio y tiempo, en ésta se observa que después del esfuerzo de preconsolidación el cambio del esfuerzo efectivo medio es casi lineal en el tiempo. Además, en la Figura 5a se observa que el suelo no había perdido toda su estructura antes de la descarga y esta estructura se preserva cuando se vuelve a cargar, como efecto se tiene un aumento del esfuerzo de fluencia en la recarga por el efecto de la cementación (el aumento del esfuerzo de fluencia es de $55 \mathrm{kPa}$ ). a)

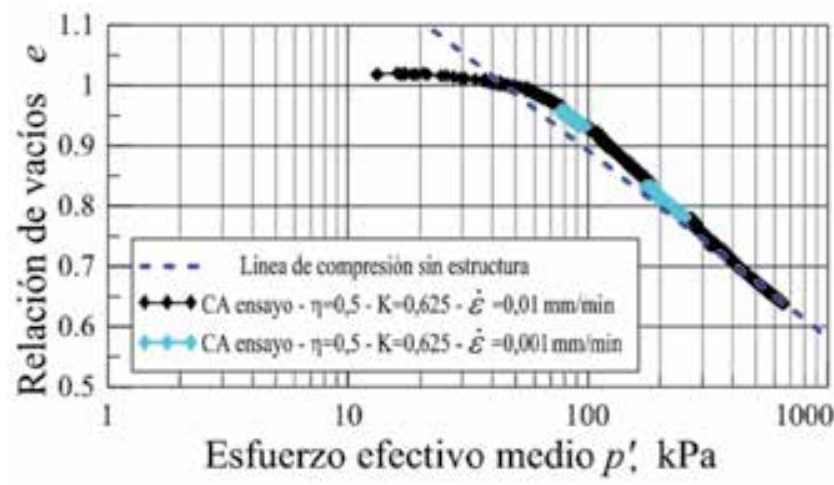

b)

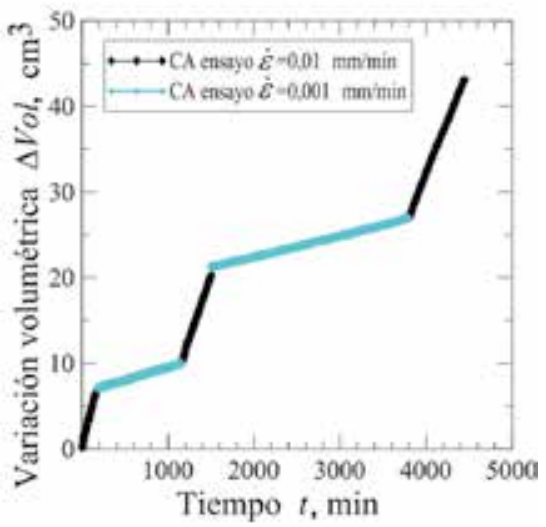

Figura 4: Ensayo de consolidación en triaxial $\operatorname{con} \eta=0.5$; a) curvas de compresibilidad y b) variación volumétrica versus tiempo.

a)

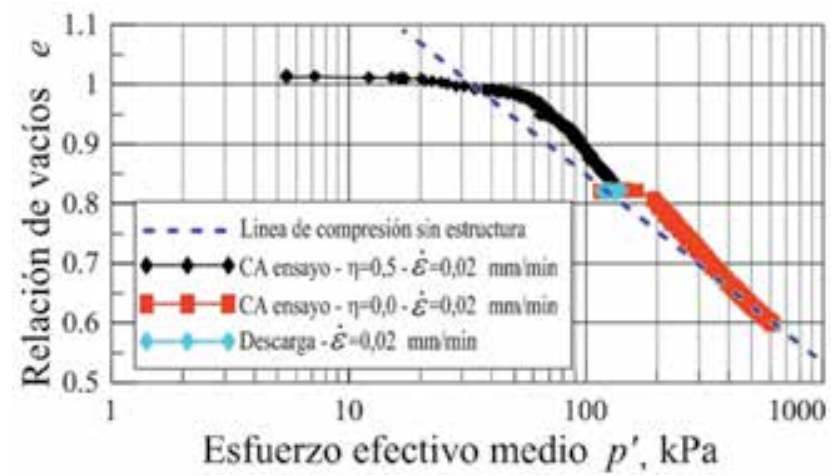

b)

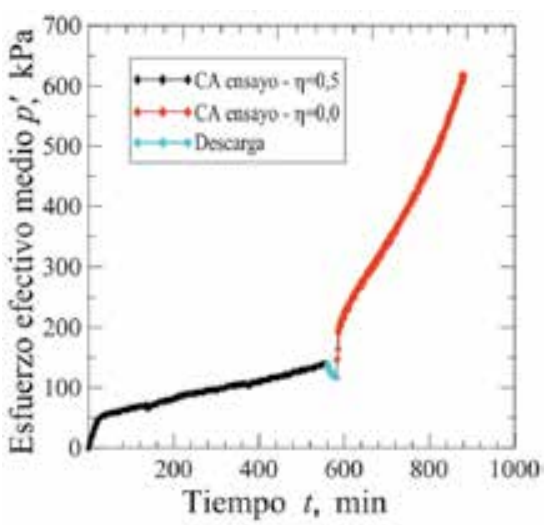

Figura 5: Ensayo de consolidación en triaxial con $\eta=0.5-0.0$; a) curvas de compresibilidad y b) esfuerzo efectivo medio versus tiempo 
Posteriormente en otras seis muestras de suelo se realizaron ensayos triaxiales bajo condiciones drenadas y no drenadas (Figura 6). Estos ensayos permiten analizar el comportamiento mecánico del suelo bajo condiciones de esfuerzos cortantes y determinar parámetros para el estado crítico y rigidez de suelo

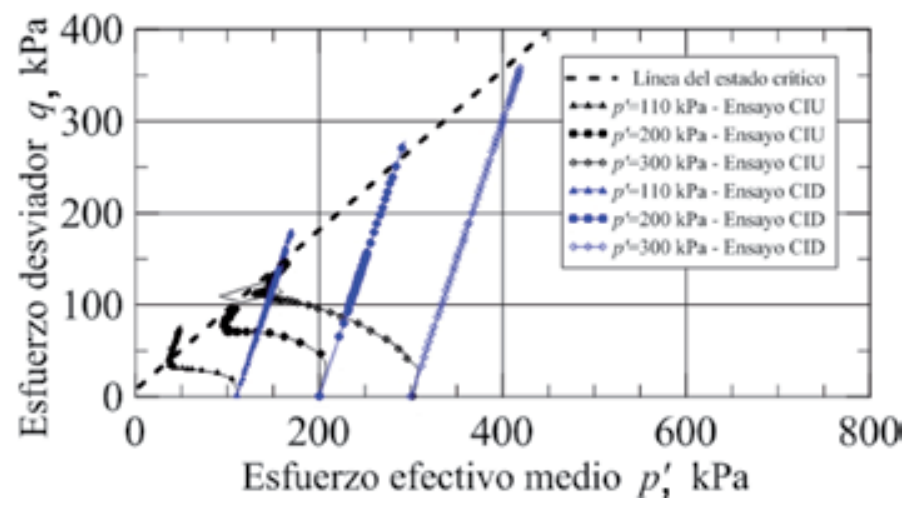

Figura 6: Trayectorias de esfuerzos en los ensayos triaxiales

Los primeros tres ensayos fueron saturados y consolidados isotrópicamente a presiones de confinamiento efectivas de 110,200 y $300 \mathrm{kPa}$. Posteriormente fue generado un esfuerzo desviador de forma no drenada (ensayo CIU) a una velocidad de deformación constante de $\dot{\varepsilon}=0.05$ $\mathrm{mm} / \mathrm{min}$. Las trayectorias de tensiones se muestran en la Figura 6. De esta figura se observa que para mayor presión de confinamiento, se aumenta el esfuerzo desviador máximo. Además, la Figura 7a muestra que crece el esfuerzo desviador a mayor esfuerzo de confinamiento a una deformación unitaria determinada. De forma análoga ocurre con el exceso de presión de poros (Figura 7b), lo cual es un comportamiento típico reportado en textos de Mecánica de Suelos (Whitlow, 2000; Helwany, 2007). Por otra parte, dos de las tres trayectorias siguen casi la misma línea de estado crítico, el ensayo con una presión de confinamiento de $110 \mathrm{kPa}$ al parecer sigue otra línea de estado crítico. Otro factor, es que en los tres ensayos siguen la línea del estado crítico y continúan por ella (Figura 6), lo que tiene como consecuencia una continua ganancia en resistencia a mayor deformación unitaria (Figura 7a). El comportamiento anterior no es común para arcilla, como lo muestra el trabajo de Roscoe et al. (1963) donde las trayectorias solo llegan a la línea de estado crítico.

Se realizaron otros tres ensayos saturados y consolidados a esfuerzos efectivos de 110, 200 y $300 \mathrm{kPa}$, pero generando esfuerzos desviadores en condiciones drenada (ensayo CID) y algunos con cambios en la velocidad de deformación vertical, para observar los efectos viscosos en el suelo.

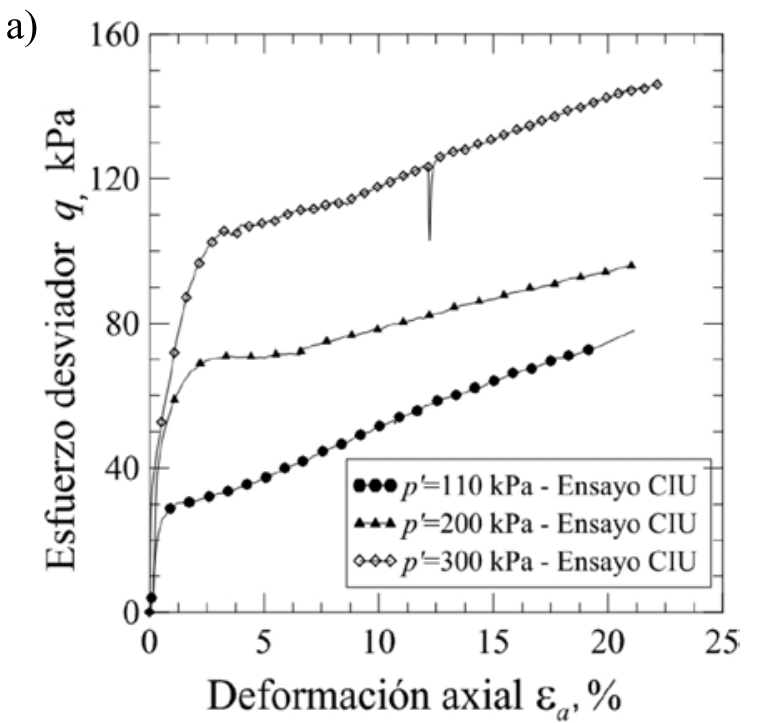

b)

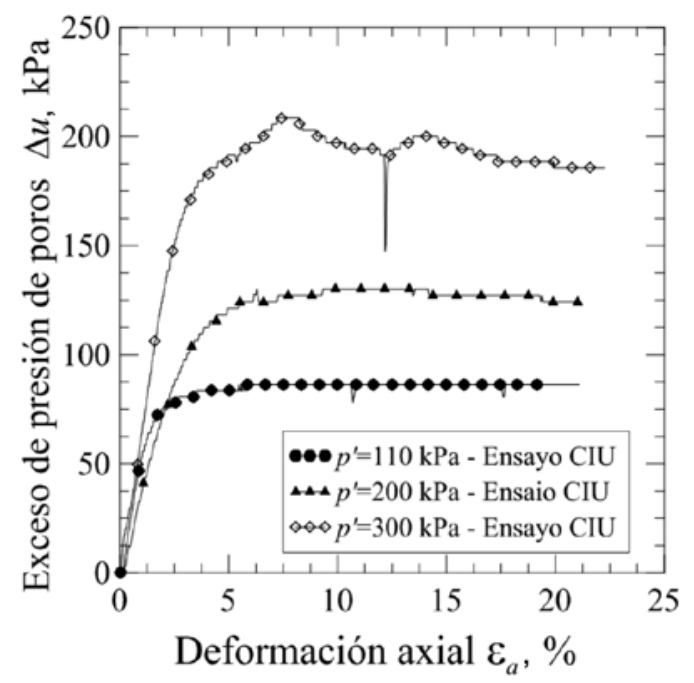

Figura 7: Ensayos triaxiales no drenados

El primer ensayo fue consolidado a un esfuerzo efectivo de $p^{\prime}=110 \mathrm{kPa}$, el cual superó el esfuerzo de preconsolidación. Posterior a la consolidación se inició el corte a una velocidad de deformación constante de $\dot{\varepsilon}=0.03 \mathrm{~mm} / \mathrm{min}$ con cambios de velocidad a $\dot{\varepsilon}=0.003 \mathrm{~mm} / \mathrm{min}$. El segundo ensayo fue realizado del mismo modo, pero consolidado a un esfuerzo efectivo medio de $200 \mathrm{kPa}$. El último ensayo fue realizado a una presión efectiva de $300 \mathrm{kPa}$, sin cambio de velocidad. De los ensayos anteriormente mostrados, se puede observar que las trayectorias llegan casi a la 
misma línea del estado crítico (Figura 6). También, se evidenció una baja influencia del cambio de velocidad en el comportamiento mecánico del suelo (Figura 8a).

a)

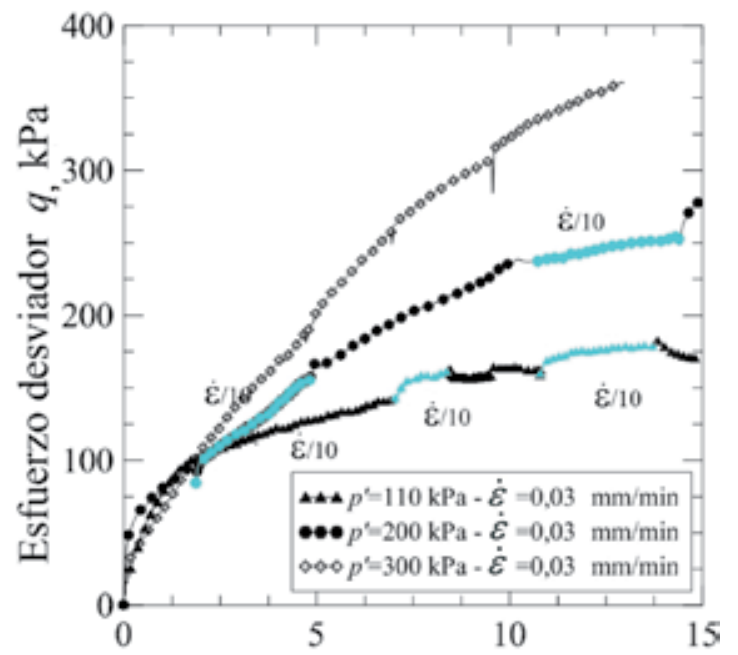

Deformación unitaria axial $\varepsilon_{a}, \%$

b)

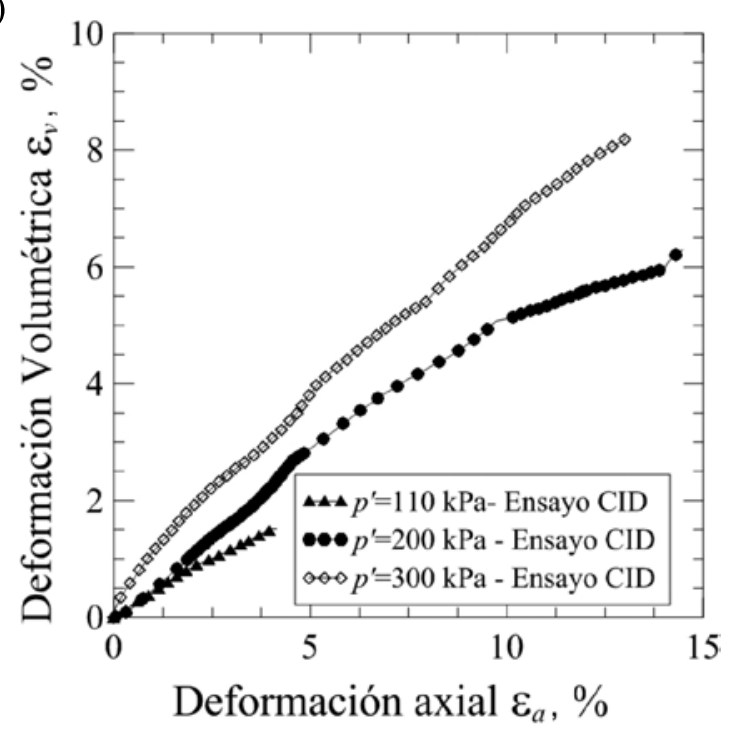

Figura 8: Ensayos triaxiales drenados.

La Figura 8b muestra la variación de deformación volumétrica con la deformación axial, donde se observa que a mayor presión efectiva confinante mayor es la deformación volumétrica. Otro aspecto importante es la presencia de cementación, similares a los reportados por Lagioia y Nova (1995) para suelos cementados. La interpretación es: a un esfuerzo efectivo medio de cámara de $110 \mathrm{kPa}$ la cementación influye en la rigidez del suelo hasta $7 \%$ de deformación unitaria axial, para $200 \mathrm{kPa}$ la influencia de la cementación en la rigidez es de 2 al $4 \%$ de deformación axial, y para $300 \mathrm{kPa}$ la influencia de la cementación en la rigidez es para una deformación menor al $1.5 \%$ de la deformación axial (Figura 8a). Esto se confirma al observar las Figuras 4, 5 y 6, donde en estos esfuerzos efectivos medios está la presencia de la cementación del suelo.

\section{Modelos constitutivos}

Los primeros modelos constitutivos usados para suelos fueron basados en la teoría de la plasticidad perfecta aplicada a los metales (Helwany, 2007). El comportamiento de los metales difiere en gran medida del comportamiento de los suelos, ya que el suelo tiene características de no homogeneidad, no linealidad y anisotropía, como lo evidencian los trabajos realizados por Roscoe et al. (1958), Burland (1990), Leroueil y Vaughan (1990), Cuccovillo y Coop (1999), Liu y Carter (2002) y Masín (2006). Para simular mejor el comportamiento esfuerzo deformación del suelo han sido creados extensiones de los modelos para metales y otros nuevos modelos (elastoplástico, hipoplásticos, viscohipoplásticos). A pesar de lo expuesto anteriormente, no se ha podido desarrollar un modelo unificado con la capacidad de representar la totalidad de características que posee el suelo y que además sea aceptado por la totalidad de la comunidad científica.

En la actualidad, los modelos constitutivos para suelos han sido extendidos para introducir los efectos de la estructura y cementación del suelo. Esta extensión fue debida a las evidencias experimentales estudiadas por Burland (1990), Leroueil y Vaughan (1990), Cuccovillo y Coop (1999) y Sorensen et al. (2007), que muestran diferencias en el comportamiento de los suelos reconstituidos y los suelos inalterados. Por esta razón varios investigadores han desarrollado nuevas formulaciones constitutivas tomando en cuenta el efecto de la estructura en el suelo. Entre las propuestas destacan las de Vatsala et al. (2001), Liu y Carter (2002), Masín (2006), Fuentes et al. (2010) y Yan y Li (2011), entre otras.

A continuación se presentan las ecuaciones y conceptos básicos para introducir el efecto de la estructura a tres modelos constitutivos, los cuales fueron escogidos por ser conocidos. Las implementaciones realizadas usan la metodología de las UMATs (sub rutina del programa Abaqus para el comportamiento del material), por medio del programa incremental driver realizado por Niemunis (2008). 


\section{Cam Clay con estructura CCS}

El modelo Cam Clay con estructura propuesto por Liu y Carter (2002) para suelos naturales, está dentro de la familia de modelos elastoplásticos Cam Clay que se desarrollaron para representar el comportamiento del suelo basados en los conceptos de estado crítico propuesto por Schofield y Wroth en la década de los 60 (Roscoe et al., 1963) y posteriormente modificado por Roscoe y Burland (1968), Cam Clay modificado CCM. Estos modelos cuentan con gran popularidad, por su simplicidad y forma gráfica de representar el comportamiento del suelo (Pedroso, 2006). Las modificaciones del modelo son:

i) Adición de la estructura a la ley de compresión isotrópica del CCM, la cual es expresada mediante la siguiente expresión:

$$
d \varepsilon^{p}=(\lambda-\kappa) \frac{d p_{y, i}^{\prime}}{(1+e) p_{y, i}^{\prime}}+b \Delta e\left(1+\frac{\eta}{M-\eta}\right) \frac{d p_{y, i}^{\prime}}{(1+e) p_{y, i}^{\prime}}
$$

donde $\eta$ es un factor que tiene en cuenta la relación de esfuerzos, $M$ es la pendiente del estado crítico del CCM, $e$ relación de vacíos actual, $\Delta e$ la relación de vacíos por la estructura, $b$ es la tasa de pérdida de estructura del suelo (parámetro nuevo del modelo con estructura) y $p_{y, i}^{\prime}$ es el esfuerzo de fluencia el cual describe el tamaño inicial de la superficie de fluencia con estructura (parámetro nuevo del modelo con estructura), como se muestra en la Figura 9.

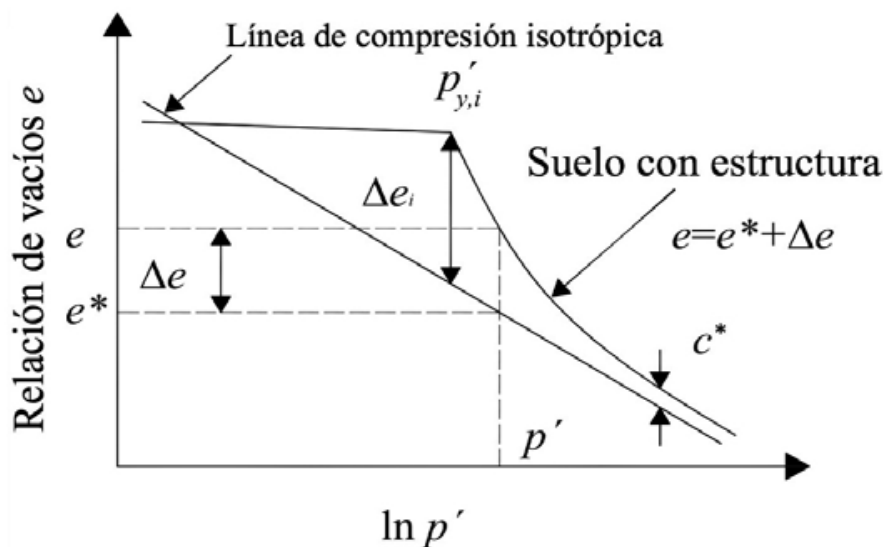

Figura 9: Idealización del comportamiento de compresión isotrópica de un suelo reconstituido y natural (Liu y Carter, 2002).

La evolución de la relación de vacíos por estructura $\Delta e$, en la cual se muestra el funcionamiento del parámetro $b$, está dada por la siguiente expresión:

$$
\Delta e=\Delta e_{i}\left(\frac{p_{y, i}^{\prime}}{p^{\prime}}\right)^{b}+c^{*}
$$

Por último, en este modelo la relación de vacíos del suelo natural $e$ es descompuesta en la relación de vacíos por estructura $\Delta e$ y una relación de vacíos reconstituida $e^{*}$, ecuación (3). La Figura 9 muestra la explicación física de los parámetros del modelo.

$e=e^{*}+\Delta e$

ii) Se cambia la regla de flujo del modelo CCM por una regla de flujo no asociada ecuación (4), por medio del factor (1- $\omega \Delta e)$, donde $\omega$ es un parámetro que influye en la regla de flujo y en las deformaciones plásticas desviadoras. La regla de flujo fue cambiada al observar que la relación entre los incrementos de deformación desviadoras y volumétricas plásticas son mayores en muestras reconstituidas (suposición CCM) que en suelos inalterados (Liu y Carter, 2002).

$$
\frac{d \varepsilon_{d}^{p}}{d \varepsilon_{v}^{p}}=\frac{2(1-\omega \Delta e) \eta}{M^{2}-\eta^{2}}
$$

A continuación se muestran las ecuaciones y definiciones del modelo de forma tridimensional para ser implementado en una plataforma de elementos finitos. El esfuerzo isotrópico $p$ ' y desviador $q$ se definen en las invariantes dadas en las ecuaciones (5) y (6), la relación entre estas dos variables se define como $\eta$, donde $\boldsymbol{\sigma}$ es el tensor de esfuerzo de Cauchy.

$$
\begin{aligned}
& p^{\prime}=\frac{1}{3} \operatorname{tr}(\boldsymbol{\sigma}), \quad q=\sqrt{\frac{3}{2}} \xi, \quad \eta=q / p^{\prime} \\
& \text { donde } \\
& \qquad \xi=\boldsymbol{\sigma}-\frac{1}{3} \operatorname{tr}(\boldsymbol{\sigma}) \mathbf{1}
\end{aligned}
$$

1 es un tensor unitario de segundo orden. La ecuación básica del modelo está dada por la siguiente expresión:

$$
\boldsymbol{\sigma}=\mathrm{C}^{e p} \boldsymbol{\varepsilon}
$$

donde $\boldsymbol{\varepsilon}$ es el tensor de deformación y $\mathrm{C}^{e p}$ es el tensor de módulos de rigidez elastoplástica que relaciona los esfuerzos con las deformaciones. El tensor elastoplástico está dividido en dos partes como se presenta a continuación: 


$$
\mathrm{C}^{e p}=\mathrm{C}^{e}-\mathrm{C}^{p}
$$

una parte elástica $\mathrm{C}^{\mathrm{e}} \mathrm{y}$ una parte plástica $\mathrm{C}^{p}$. También se tiene la inclusión de la regla de flujo no asociada por medio de las derivadas respecto a la función potencial plástico $g$, las cuales son mostradas en el Apéndice.

$$
\begin{aligned}
& \mathrm{C}^{e}=K 1 \otimes 1+2 \mu\left(\mathbf{I}-\frac{1}{3} 1 \otimes 1\right) \\
& \mathrm{C}^{p}=\frac{\mathrm{C}^{e}: \frac{d f}{d \boldsymbol{\sigma}} \otimes \frac{d g}{d \boldsymbol{\sigma}}: \mathrm{C}^{e}}{\frac{d f}{d \boldsymbol{\sigma}}: \mathrm{C}^{e}: \frac{d g}{d \boldsymbol{\sigma}}-\left(\frac{d f}{d \boldsymbol{\varepsilon}_{v}^{p}} \frac{d g}{d p}\right)}
\end{aligned}
$$

La regla de endurecimiento está en un espacio semilogarítmico del esfuerzo efectivo medio y el volumen específico, donde el aumento de las deformaciones cambia el esfuerzo como se muestra en la siguiente ecuación:

$$
\dot{p}_{y, i}^{\prime}=p_{y, i}^{\prime} \vartheta \dot{\varepsilon}_{v}^{p}
$$

donde el factor $\vartheta$ es función de la ley de compresión del material por medio de la relación de vacíos $e$ y los parámetros $\kappa$ y $\lambda$,

$$
\vartheta=\frac{1+e}{\lambda-\kappa}
$$

La variación de la deformación plástica volumétrica viene dada por:

$$
\dot{\varepsilon}_{v}^{p}=\operatorname{tr}\left(\dot{\boldsymbol{\varepsilon}}^{p}\right)
$$

El incremento de las deformaciones plásticas tiene la siguiente regla de flujo no asociada.

$$
d \varepsilon^{p}=\dot{\phi} \frac{d g}{d \boldsymbol{\sigma}}
$$

donde $g$ es la función de potencial plástico y $\dot{\phi}$ es el multiplicador plástico, definido al igual que en el modelo CCM, como se muestra en la siguiente ecuación:

$$
\dot{\phi}=\frac{\frac{d f}{d \boldsymbol{\sigma}}: \mathrm{C}^{e}: \boldsymbol{\varepsilon}}{\frac{d f}{d \boldsymbol{\sigma}}: \mathrm{C}^{e}: \frac{d g}{d \boldsymbol{\sigma}}-\left(\frac{d g}{d \varepsilon_{v}^{p}} \frac{d g}{d p}\right)}
$$

Una de las principales características del modelo elastoplástico es la superficie de fluencia. Esta muestra el punto donde empiezan las deformaciones elastoplásticas en una trayectoria de esfuerzos. La ecuación matemática que define dicha superficie es:

$$
f=M^{2} p^{\prime}\left(p^{\prime}-p_{y, i}^{\prime}\right)+q^{2}
$$

Por último, las operaciones tensoriales utilizadas y las derivadas respecto a la función $f$ se encuentran en el Apéndice. Los parámetros del modelo están divididos en dos parte: 1) parámetros intrínsecos del material iguales al modelo CCM: $\lambda$ у к son las pendientes de la rama virgen y la rama de descarga de la curva de consolidación del suelo, $K$ es el módulo volumétrico, $N$ es el volumen específico a un esfuerzo efectivo medio de $1 \mathrm{kPa}$ y $M$ es la pendiente del estado crítico en el plano $p^{\prime}-q$; 2) parámetros por la estructura, los cuales son tres: el primero es la tasa de pérdida de estructura del suelo $b$; el segundo parámetro es $\omega$ el cual influye en la regla de flujo; y el último es el esfuerzo de fluencia con estructura $p_{y, i^{\prime}}^{\prime}$

Por último, las derivadas respecto a la función $f$ y la definición de las operaciones tensoriales utilizadas se encuentran en el Apéndice. Se verificó la implementación del modelo con simulaciones encontradas en la literatura realizadas por Liu y Carter (2006). La primera simulación ejecutada fue una compresión triaxial sobre una muestra de Calcarenita realizada por Lagioia y Nova (1995) y mostrada en la Figura 10 y la segunda simulación es de un triaxial drenado con una presión confinante de $2000 \mathrm{kPa}$ en el mismo suelo (Figura 11). De las simulaciones realizadas de los ensayos, se puede afirmar que la implementación realizada por Liu y Carter (2002) y la del presente trabajo obtienen resultados similares (Figuras 10 y 11).

\section{Modelo hipoplástico con estructura HPS}

El marco teórico de la Hipoplasticidad fue desarrollado por Kolymbas (1977) y definida con una rigidez tangencial que sea una función continua de la velocidad de deformación (Niemunis, 2003). Posteriormente Kolymbas realiza la formulación del un modelo hipoplástico y desde entonces se han presentado varias modificaciones al modelo como las de Wu (1992), Wolffersdorff (1996) y Niemunis (2003) 
a)

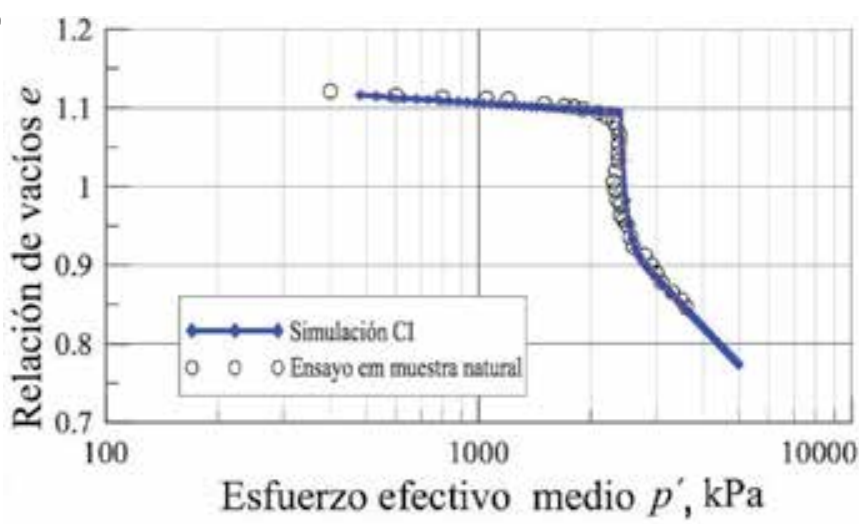

b)

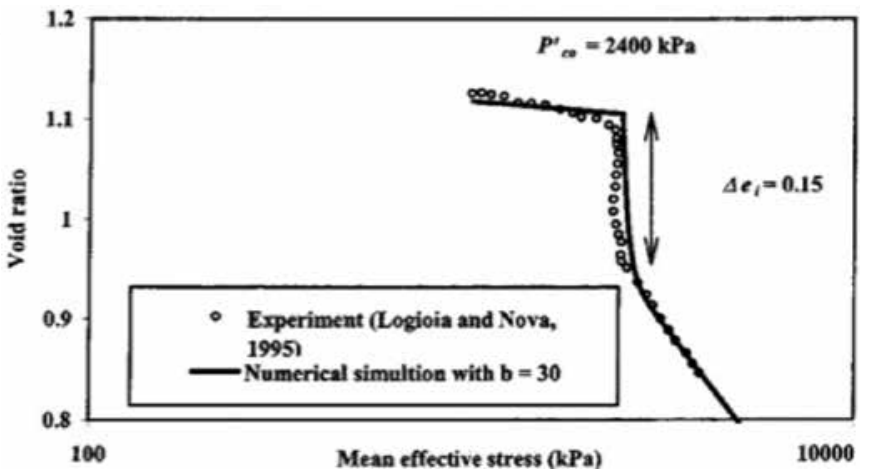

Figura 10: a) Implementación realizada y b) implementación realizada por Liu y Carter (2006)

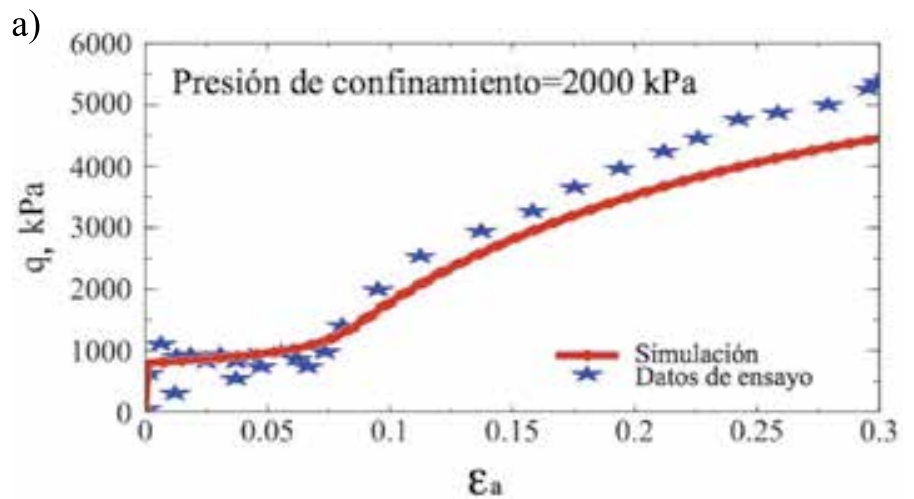

b)

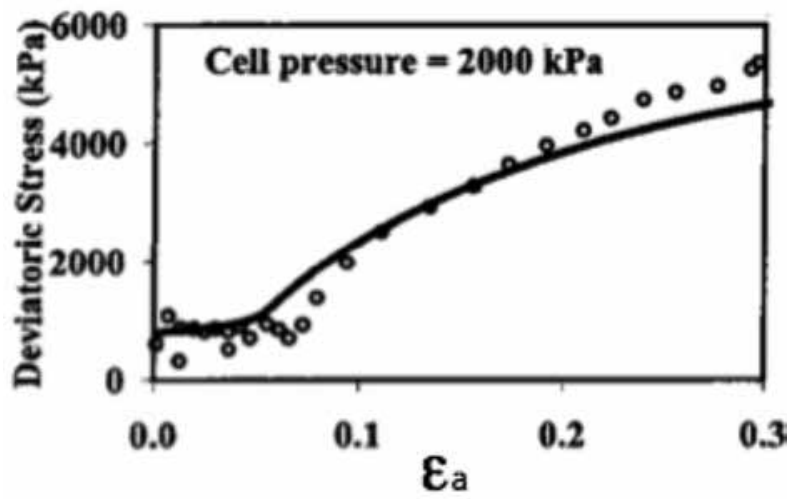

Figura 11: a) Implementación realizada y b) implementación realizada por Liu y Carter (2006) entre otros. Los anteriores modelos fueron propuestos para suelos granulares, sin embargo se han presentado extensiones para representar el comportamiento de suelos finos como las propuestas por Niemunis (2003) y Masín (2006) para suelos inalterados (con estructura).

La modificación realizada al modelo es la incorporación de la estructura a través de la propuesta hecha por Baudet y Stallebras (2004) y modificada por Masín (2006). La propuesta consiste en la incorporación de un tamaño mayor de la superficie de estado limite SBS, a través de la alteración del esfuerzo equivalente de Hvorslev por un escalar $s$ como se ilustra en la Figura 12. Para la modificación se agregan 3 nuevos parámetros $\left(s_{0}, k, A\right)$, donde $s_{0}$ es el valor inicial de la variable de estado $s$ (factor de estructura o sensibilidad) mostrada en la ecuación (19) (ley de degradación). El factor $s_{f}$ es el límite a un estado estable (Masín, 2006) con un valor de 1 (Figura 12). El factor $k$ es un parámetro que controla la degradación de la estructura; $\lambda^{*}$ es la pendiente de compresibilidad isotrópica virgen en doble logaritmo natural; $\dot{\varepsilon}^{d}$ en la ecuación (20) es llamada tasa de deformación de daño, la cual depende de las tasas de deformaciones volumétricas y cortantes, en la cual se incluye el último factor adicionado $A$, el cual controla la importancia de las deformaciones cortantes con $0<A<0.5$ (Masín, 2006).

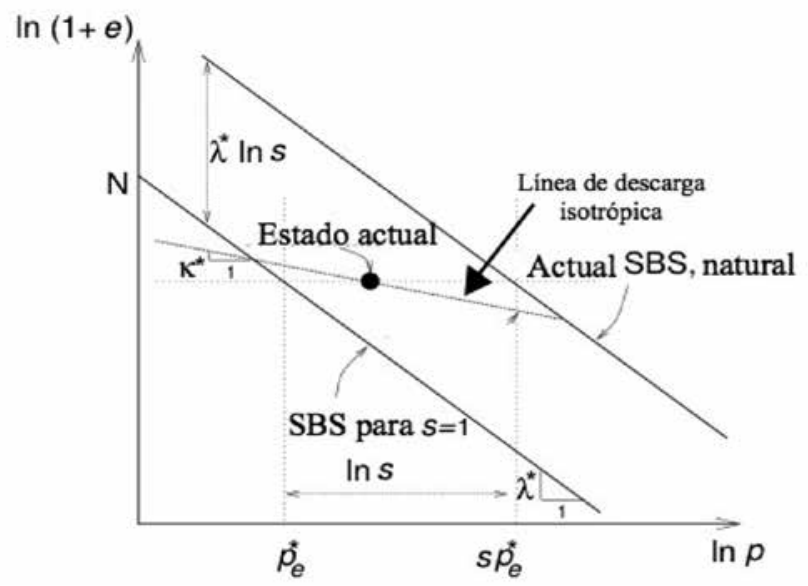

Figura 12: Idealización del comportamiento de compresibilidad de un suelo reconstituido y natural (Masín, 2006)

$$
\dot{s}=-\frac{\kappa}{\lambda^{*}}\left(s-s_{f}\right) \dot{\varepsilon}^{d}
$$

$$
\dot{\varepsilon}^{d}=\sqrt{\dot{\varepsilon}_{v}^{2}+\frac{A}{1-A} \dot{\varepsilon}_{s}^{2}}
$$


A continuación se mostrarán las principales características del modelo para ser implementado en 3D. La ecuación básica se muestra a continuación.

$$
\stackrel{\circ}{\mathbf{T}}=f_{s} \mathcal{L}: \mathbf{D}+f_{s} f_{d} \mathbf{N}\|\mathbf{D}\|
$$

Definiendo T como la tasa de cambio del tensor de esfuerzo de Cauchy en el tiempo, D como la tasa de cambio de las deformaciones en el tiempo, el tensor de cuarto orden $\mathcal{L}$ y el tensor de segundo orden $\mathbf{N}$.

$$
\mathcal{L}=\frac{1}{\hat{\mathbf{T}}: \hat{\mathbf{T}}}\left(c_{1} F^{2} \mathbf{I}+c_{2} a^{2} \hat{\mathbf{T}} \otimes \hat{\mathbf{T}}\right)
$$

$\mathcal{L}$ es un tensor constitutivo de cuarto orden en función del tensor de esfuerzos de Cauchy T, II es un tensor unitario de cuarto orden y $F$ es el criterio de estado crítico de Matsuoka-Nakai dado por:

$$
F=\sqrt{\frac{1}{8} \tan ^{2} \psi+\frac{2-\tan ^{2} \psi}{2+\sqrt{2} \tan \psi \cos 3 \theta}}-\frac{1}{2 \sqrt{2}} \tan \psi
$$

El factor escalar $a$ está dado por:

$$
a=\frac{\sqrt{3}\left(3-\sin \varphi_{c}\right)}{2 \sqrt{2} \sin \varphi_{c}}
$$

en que $\varphi_{c}$ es el ángulo de fricción en estado crítico. En los factores $c_{1}, c_{2} \mathrm{y} \alpha$ está la ley de compresión del material y se presentan a continuación:

$$
c_{1}=\left(\frac{2\left(3+a^{2}-2^{\alpha} \sqrt{3} a\right)}{9 r S_{i}}\right)
$$

En que $r$ es una constante que relaciona el módulo volumétrico con el módulo cortante no drenado.

$$
\begin{gathered}
c_{2}=1+\left(1-c_{1}\right) \frac{3}{a^{2}} \\
\alpha=\frac{1}{\ln 2} \ln \left[\frac{\lambda^{*}-\kappa^{*} S_{i}}{\lambda^{*}+\kappa^{*} S_{i}}\left(\frac{3+a^{2}}{a \sqrt{3}}\right)\right]
\end{gathered}
$$

Además, se tiene en cuenta la estructura con el factor $S_{i}$ que modifica la barotropía del material y que multiplica los parámetros $r, \kappa^{*}$ para la preservación del sentido físico de estos parámetros en el modelo (Masín, 2006).

$$
S_{i}=\frac{s-\left(s-s_{f}\right)}{s}
$$

El tensor $\mathbf{N}$ se expresa como:

$$
\mathbf{N}=\mathcal{L}:-Y\left(\frac{\mathbf{m}}{\|\mathbf{m}\|}\right)
$$

donde el tensor $\mathbf{m}$ corresponde a la regla de flujo del material, expresada como:

$$
\mathbf{m}=-\frac{a}{F}\left[\hat{\mathbf{T}}+\hat{\mathbf{T}}^{*}-\frac{\hat{\mathbf{T}}}{3}\left(\frac{6 \hat{\mathbf{T}}: \hat{\mathbf{T}}-1}{\left.\left(\frac{a}{F}\right)^{2}+\hat{\mathbf{T}}: \hat{\mathbf{T}}\right)}\right)\right]
$$

En $Y$ está el criterio de estado crítico en función de las invariantes del tensor de esfuerzos.

$$
Y=\left(\frac{\sqrt{3} a}{3+a^{2}}-1\right)\left[\frac{\left(I_{1} I_{2}+9 I_{3}\right)\left(1-\sin ^{2} \varphi_{c}\right)}{8 I_{3} \sin ^{2} \varphi_{c}}\right]
$$

Para terminar los componentes de la ecuación se tienen los factores escalares $f_{s}$ y $f_{d}$, que representan los factores de barotropía y picnotropía del material.

$$
\begin{gathered}
f_{s}=S_{i} \frac{\operatorname{tr} \mathbf{T}}{\lambda^{*}}\left(3+a^{2}-2^{\alpha} a \sqrt{3}\right)^{-1} \\
f_{d}=\left(\frac{2 p}{s p_{e}^{*}}\right)^{\alpha}
\end{gathered}
$$

Los parámetros usados por el modelo son 8. Los parámetros $s, k, A$ que representan la estructura del material ya fueron explicados. Los 5 restantes pueden ser obtenidos de una muestra reconstituida o inalterada a través de una consolidación triaxial isotrópica, donde se obtienen 3 parámetros $\left(\lambda^{*}, \kappa^{*}, N\right)$ al igual que en el modelo Cam Clay. Pero con la diferencia que son obtenidos en un plano de ejes en escala de logaritmo natural. De un ensayo de corte triaxial no drenado se obtiene $r$ que relaciona el módulo volumétrico con el módulo cortante no drenado, y el ángulo $\varphi_{c}$ el cual es análogo a $M$ que es la pendiente del estado crítico del modelo Cam Clay. 
Se realizaron simulaciones para comprobar la implementación por medio de un ensayo de compresión triaxial (Figura 13a) y ensayos de corte triaxial drenados en arcilla de Marts (Figura 14a). Estas simulaciones son comparadas con la simulaciones realizadas por Fuentes et al. (2010) en las Figuras $13 \mathrm{~b}$ y $14 \mathrm{~b}$ para un modelo Viscohipoplástico con estructura, que es una variación del modelo Hipoplástico y posee la misma ley de estructura del material.

\section{Subloading Cam Clay SLCC}

El modelo Subloading Cam Clay es propuesto por Pedroso (2006) tomando como base el trabajo presentado por Nakai y Hinokio (2004) y extendido para representar estructura con la propuesta realizada por Nakai et al. (2009). Este modelo es una modificación del CCM que fue desarrollado para suelos reconstituidos. Las modificaciones del modelo son descritas a continuación: la primera modificación es la introducción de la variable de estado $\rho$ que representa la preconsolidación del suelo. Esta variable es la diferencia entre la relación de vacíos actual $e$ preconsolidada y la relación de vacíos en la línea normal de consolidación $e_{N}$ para un mismo esfuerzo, como se muestra en la Figura 15b. Ahora, en la Figura 15a en el espacio de esfuerzos se observan dos superficies, una es la superficie normal del CCM para el esfuerzo máximo al que ha estado sometido el suelo $p_{l e}$ y la otra superficie de menor tamaño es para el estado actual de esfuerzos $p_{l}$, la cual es llamada superficie de subcarga. La distancia $\delta=p_{l e}-p_{1}$ es la esencia del concepto de subcarga que es análoga a $\rho$ (Giraldo y Farias, 2011). Lo anterior, tiene inferencia en el paso del estado preconsolidado al estado normalmente consolidado de una forma suavizada y no de la forma discontinua como en el modelo CCM se pasa de un estado a otro. Esto se realiza por medio de una función $G$ que hace que $\rho$ evolucione de forma parabólica (Figura 16a) con la influencia del parámetro $c$, según la expresión:

$$
G(\rho)=c \rho|\rho|
$$

La segunda modificación del modelo es la inclusión de la estructura por medio de la variable de estado $\omega$ que presenta un comportamiento lineal (Figura 16b) respecto a la función $Q$ por medio del parámetro $b^{\prime}$ :

$$
Q(\omega)=b^{\prime} \omega
$$

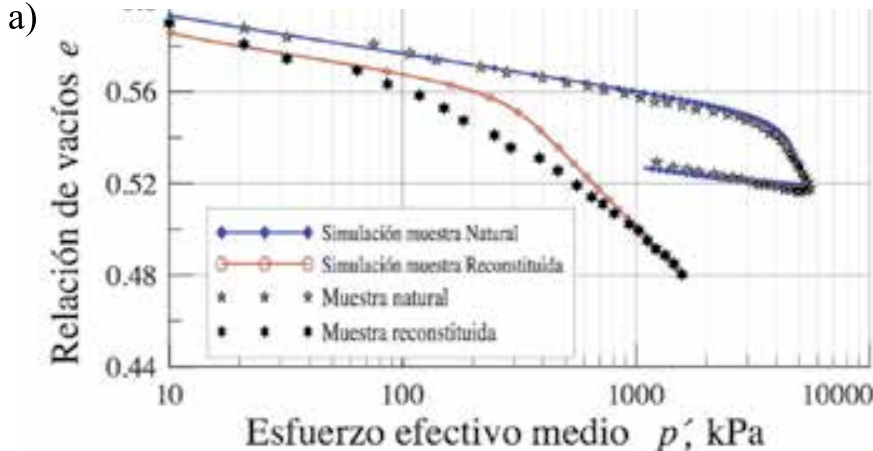

b)

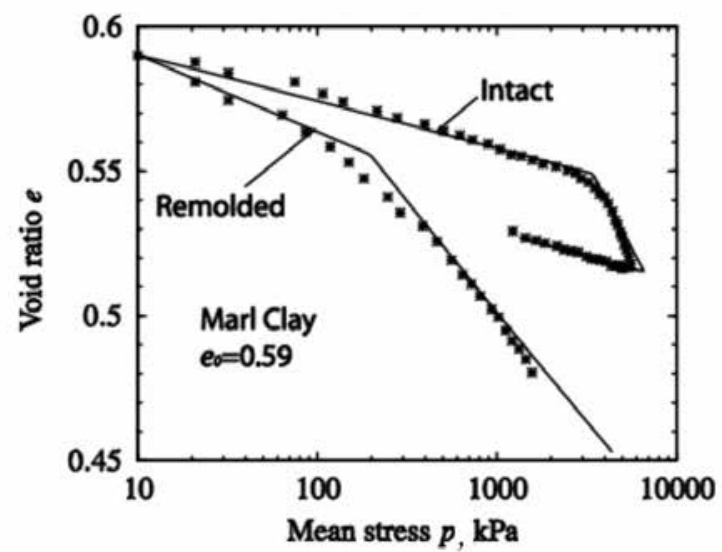

Figura 13: a) Implementación realizada y b) implementación de Fuentes et al. (2010)

a)

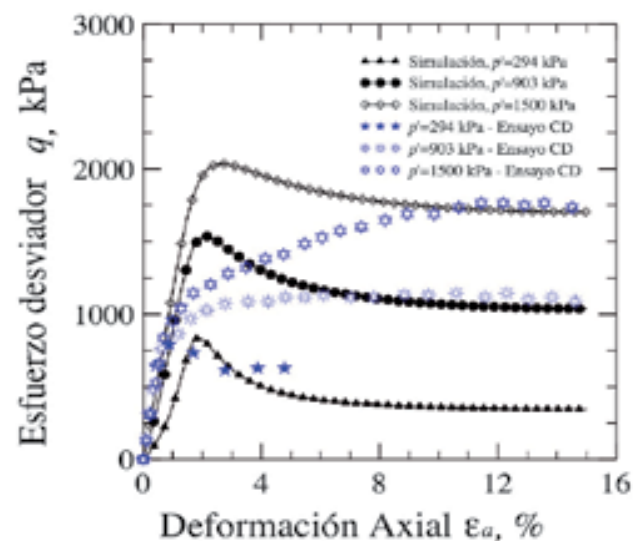

b)

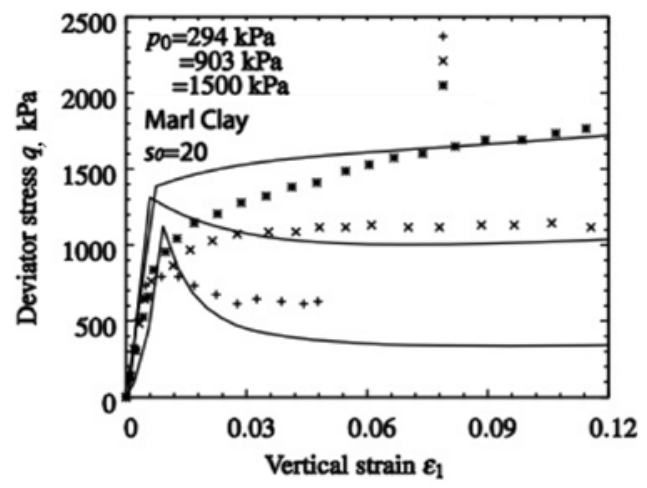

Figura 14: a) Implementación realizada y b) implementación de Fuentes et al. (2010) 
a)

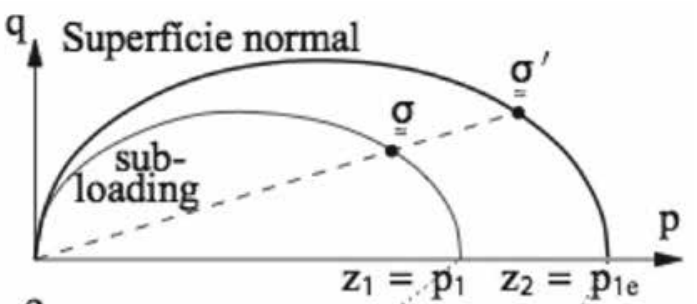

b)

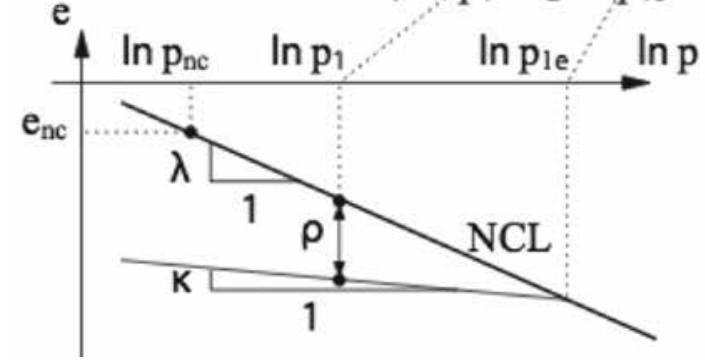

Figura 15: a) Superficie de subcarga y b) medida de $r$ (Farias et al., 2009). a)

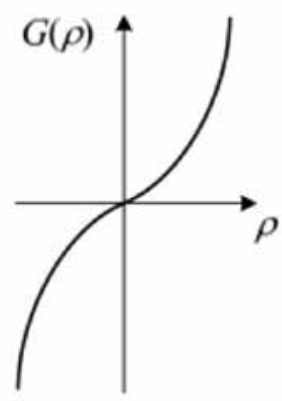

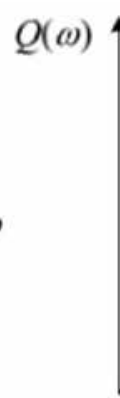

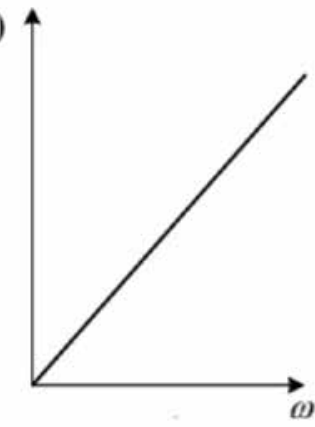

b)

Figura 16: Evolución de las variables de estado $\rho$ y $\omega$ con las funciones $\mathrm{G}(\rho)$ y $\mathrm{Q}(\omega)$

Este concepto es concebido afectando $\rho$ con una densidad imaginaria adicional que hace la obtención de una densidad negativa - $\rho$ que representa la estructura del suelo. Esto ocasiona que la línea de compresión del suelo pase por arriba de la línea de consolidación normal NC, para después volver a caer en ésta. La última modificación del modelo es la inclusión de la anisotropía de esfuerzos de compresión y tensión, donde la máxima relación de esfuerzos $M$ es función del ángulo de Lode (Sheng et al., 2000). Estas variables son dadas por:

$$
\begin{gathered}
M=\frac{6 \sin \varphi_{c r}}{3-\sin \varphi_{c r}} \\
\theta=\frac{1}{3} \sin ^{-1}\left(\frac{2 \sqrt{2} \operatorname{det}(\xi)}{\sqrt{3} \xi^{3}}\right)
\end{gathered}
$$

La relación de esfuerzos en función del ángulo de Lode se muestra a continuación:

$$
M(\theta)=M\left(\frac{2 \omega}{1+\omega-(1-\omega) \sin 3 \theta}\right)^{\frac{1}{4}}
$$

En que

$$
\omega=\frac{3-\sin \varphi_{c r}}{3+\sin \varphi_{c r}}
$$

De lo anterior se llega a una superficie de fluencia igual a la presentada en (18), pero con el cambio de $M$ por $M(\theta)$ y $p_{y, i}$ por $p_{1}$. A continuación se dan las ecuaciones para implementar el modelo, intentando no volver a escribir las ecuaciones dadas en CCS. La ecuación básica del modelo es igual que la del modelo CCS, ver (9). El tensor de módulos elástoplásticos $C^{\text {ep }}$ en (10) está influenciado por la densidad y la estructura en la parte plástica descrita en (40) por medio de la función $L$ en (41). Otra diferencia es que este modelo tiene una regla de flujo asociadas $(g=f)$.

$$
\begin{gathered}
\mathrm{C}^{p}=\frac{\mathrm{C}^{e}: \frac{d f}{d \boldsymbol{\sigma}} \otimes \frac{d g}{d \boldsymbol{\sigma}}: \mathrm{C}^{e}}{\frac{d f}{d \boldsymbol{\sigma}}: \mathrm{C}^{e}: \frac{d g}{d \boldsymbol{\sigma}}-\left(\frac{d f}{d \varepsilon_{v}^{p}} \frac{d g}{d p}+L\right)} \\
L=\frac{1}{\left(1+e_{0}\right)}\left(\frac{G}{p}+\frac{Q}{p}\right)
\end{gathered}
$$

La regla de endurecimiento (42) fue modificada adicionándole una parte por la subcarga y la estructura, por medio de una tasa de deformación adicional $\dot{\varepsilon}_{v}^{p(s y)}$ en (43). Esta tasa de deformación es el producto del multiplicador plástico $\dot{\phi}$ en (44) por el escalar $L$ en (41).

$$
\begin{gathered}
\dot{p}_{1}=p_{1} \vartheta\left(\dot{\varepsilon}_{v}^{p}+\dot{\varepsilon}_{v}^{p(s y)}\right) \\
\dot{\varepsilon}_{v}^{p(s y)}=\dot{\phi} L
\end{gathered}
$$

$\dot{\phi}=\frac{\frac{d f}{d \boldsymbol{\sigma}}: \mathrm{C}^{e}: \boldsymbol{\varepsilon}}{\frac{d f}{d \boldsymbol{\sigma}}: \mathrm{C}^{e}: \frac{d g}{d \boldsymbol{\sigma}}-\left(\frac{d g}{d \varepsilon_{v}^{p}} \frac{d g}{d p}+L\right)}$

Los parámetros usados por el modelo son siete. Los parámetros nuevos del modelo son $c, b$ y ya fueron explicados. Los cinco parámetros restantes son los del modelo Cam Clay modificado y fueron explicados en el 
modelo CCS y HPS para el ángulo de fricción crítico. Para la comprobación de la implementación fueron hechas comparaciones con las simulaciones de Giraldo y Farias (2011) de un ensayo de consolidación para observar el aumento de la estructura con el aumento de la variable $\omega$ (Figura 17). En la Figura 18 se presenta la comparación de un ensayo triaxial cíclico drenado en la arcilla de Fujinomori simulado por Pedroso (2006).

a)

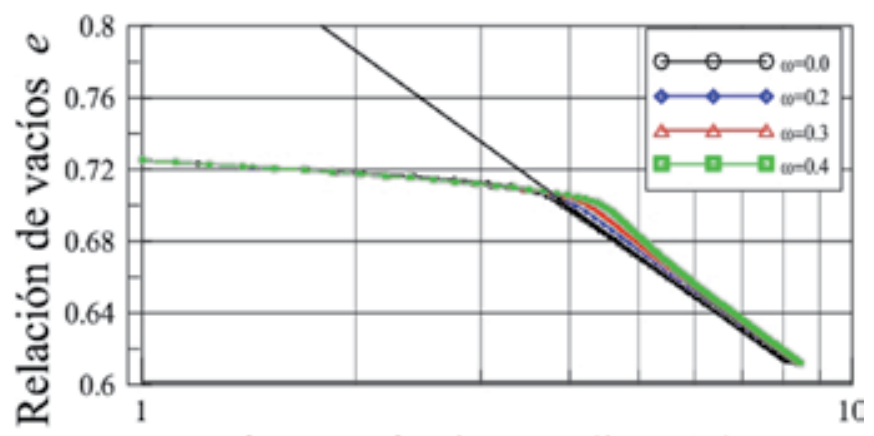

Esfuerzo efectivo medio $p^{\prime}, \mathrm{kPa}$

b)

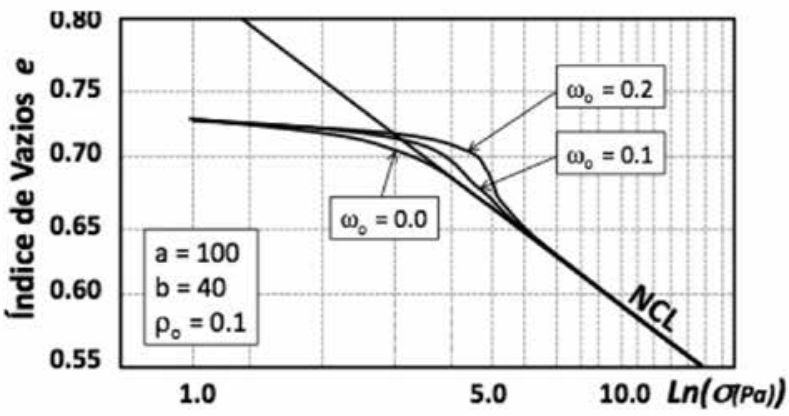

Figura 17: a) Implementación realizada y b) implementación de Giraldo y Farias (2011)

\section{Resultados}

Uno de los objetivos de esta investigación es entender cuál modelo puede representar mejor el comportamiento mecánico de la arcilla de la ciudad de Brasilia. Lo anterior se hizo por medio de la simulación de ensayos con diferentes modelos constitutivos aplicados a un punto (element tests) implementados para posteriormente debatir cual podría ser la mejor opción para predecir el comportamiento del suelo. Con los modelos constitutivos implementados en la sección anterior se realizaron simulaciones de compresiones anisotrópicas con las relaciones de esfuerzos mostradas en la sección de ensayos de laboratorio. En la Tabla 1 se presentan los parámetros obtenidos de las simulaciones realizadas en este trabajo.

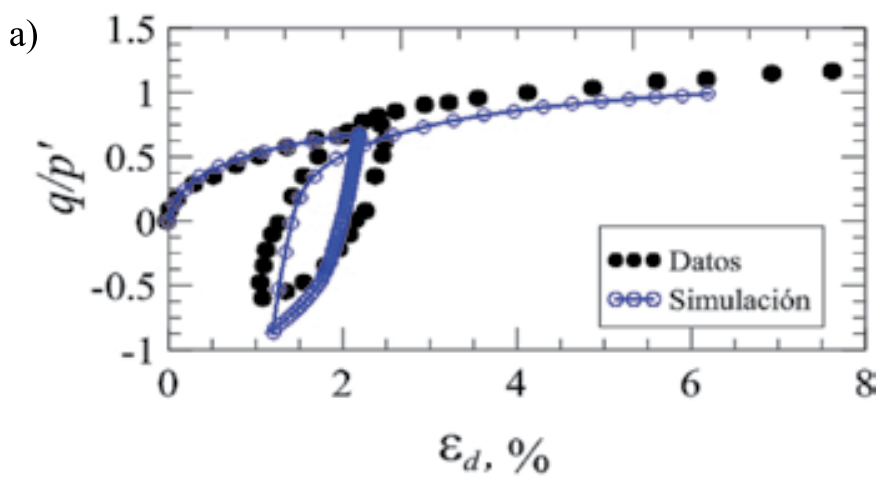

b)

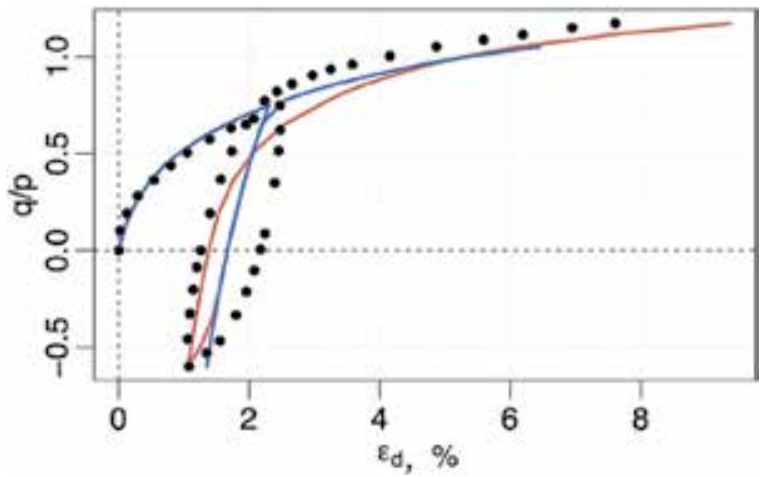

Figura 18: a) Implementación realizada y b) implementación de Pedroso (2006)

Tabla 1: Valores de parámetros obtenidos de las simulaciones

\begin{tabular}{|c|c|c|c|}
\hline Modelo & Parámetro & Valor & Observación \\
\hline \multirow{8}{*}{$\begin{array}{c}\text { Cam Clay con } \\
\text { estructura }\end{array}$} & $\kappa$ & 0.0136 & Intrínseco $^{1}$ \\
\hline & $\lambda$ & 0.164 & Intrínseco $^{1}$ \\
\hline & $p_{y, i}$ & 80 & Estructura $^{2}$ \\
\hline & $M$ & 1.43 & Intrínseco $^{1}$ \\
\hline & $\mu$ & 0.25 & Intrínseco $^{1}$ \\
\hline & $b^{\prime}$ & 30 & Estructura $^{2}$ \\
\hline & $\omega$ & 3.33 & Estructura $^{2}$ \\
\hline & $\Delta e$ & 0.015 & Estructura $^{2}$ \\
\hline \multirow{8}{*}{$\begin{array}{l}\text { Hipoplasticidad } \\
\text { con estructura }\end{array}$} & $\kappa^{*}$ & 0.0022 & Intrínseco $^{1}$ \\
\hline & $\lambda^{*}$ & 0.060 & Intrínseco $^{1}$ \\
\hline & $N$ & 2.134 & Intrínseco $^{1}$ \\
\hline & $\varphi_{c}$ & 31 & Intrínseco $^{1}$ \\
\hline & $r$ & 0.4 & Intrínseco $^{1}$ \\
\hline & $S$ & 1.75 & Estructura $^{2}$ \\
\hline & $A$ & 0.4 & Estructura $^{2}$ \\
\hline & $k$ & 2.5 & Estructura $^{2}$ \\
\hline \multirow{7}{*}{$\begin{array}{c}\text { Subloading Cam } \\
\text { Clay }\end{array}$} & $\kappa$ & 0.0115 & Intrínseco $^{1}$ \\
\hline & $\lambda$ & 0.15 & Intrínseco $^{1}$ \\
\hline & $N$ & 2.03 & Intrínseco $^{1}$ \\
\hline & $\varphi_{c}$ & 30.4 & Intrínseco $^{1}$ \\
\hline & $\mu$ & 0.25 & Intrínseco $^{1}$ \\
\hline & $b$ & 50 & Estructura $^{2}$ \\
\hline & $c$ & 12000 & Estructura $^{2}$ \\
\hline
\end{tabular}

${ }^{1}$ Parámetros provenientes del modelo inicial, los cuales pueden ser de muestras naturales o reconstituidas

${ }^{2}$ Nuevos parámetros adicionados por la estructura del suelo y obtenidos de muestras inalteradas 
La simulación del ensayo de consolidación fue realizada con una relación de esfuerzos $\eta=0.3$ y a una velocidad de deformación constante $\dot{\varepsilon}=0.03 \mathrm{~mm} / \mathrm{min}$. Durante el ensayo se simuló una relajación a una tensión efectiva media de $220 \mathrm{kPa}$ por $1200 \mathrm{~min}$ (tiempo usado en el ensayo para la estabilización del esfuerzo efectivo medio). Posteriormente se continuó con la velocidad de deformación anterior hasta un esfuerzo efectivo medio de $550 \mathrm{kPa}$ aproximadamente. Después se realizó una descarga hasta un esfuerzo efectivo medio de $5 \mathrm{kPa}$ y por último se volvió a cargar hasta $580 \mathrm{kPa}$. En las Figuras $19 \mathrm{a}$ y $19 \mathrm{~b}$ se presentan los resultados de las simulaciones realizadas en los planos de esfuerzo efectivo medio, índice de vacíos y tiempo. De estas simulaciones se observó que ninguna fue capaz de representar el "loop" presentado en el ensayo cuando se carga y descarga el suelo, lo cual puede ser importante ya que el material está sometido a cargas cíclicas. Además ninguno de los modelos representó la pérdida de esfuerzo efectivo medio en el tiempo con una deformación constante (relajación). Lo anterior es debido a que ninguno de los modelos implementados tiene en cuenta la viscosidad del suelo.

a)

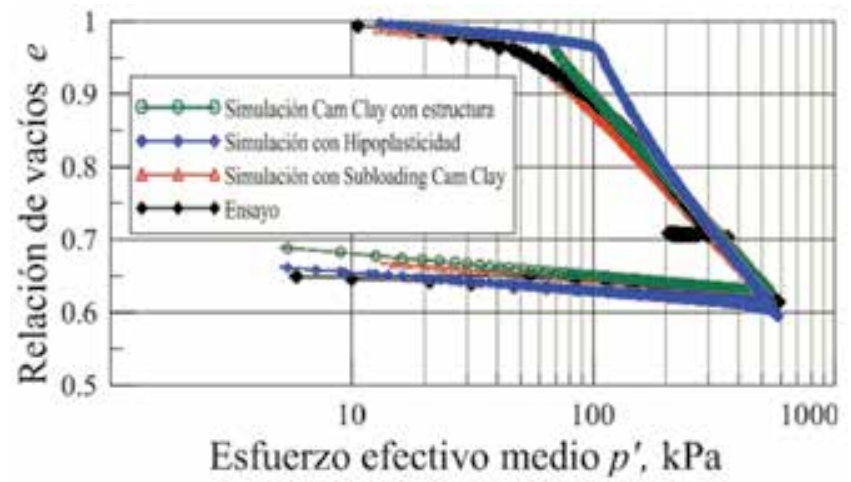

b)

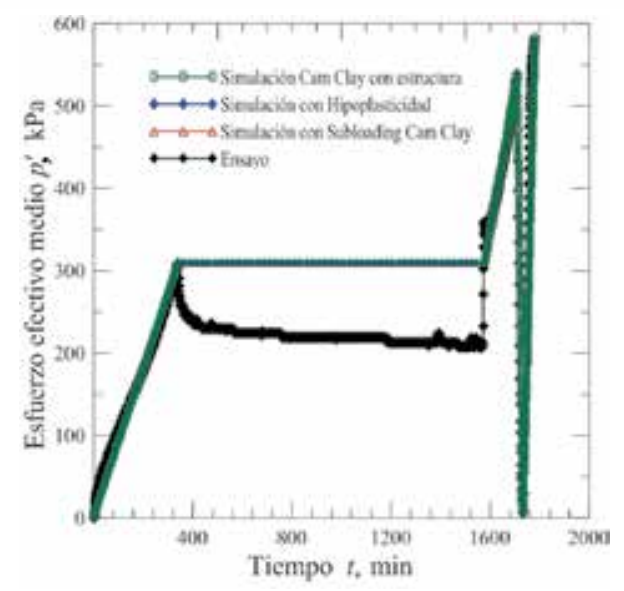

Figura 19: Comparación de simulaciones realizadas y el ensayo de consolidación con $\eta=0.3$
Se simuló un ensayo de compresión anisotrópico con una relación de esfuerzos de $\eta=0.5$. En las Figuras 20a y $20 \mathrm{~b}$ se presentan los resultados de las simulaciones y el ensayo. La trayectoria de esfuerzos está en la Figura 2. Las simulaciones fueron realizadas simulando los cambios de velocidad realizados en el ensayo de laboratorio con cambios del tiempo de simulación para una deformación unitaria dada (Figura 20b). De las anteriores simulaciones se observó que el modelo Cam Clay con estructura presentó un cambio brusco de estado preconsolidado a normalmente consolidado, el modelo Hipoplástico presentó un punto intermedio entre los tres modelos y el modelo Subloading Cam Clay presentó la transición más suave y parecida al ensayo, de un estado preconsolidado a uno normalmente consolidado.

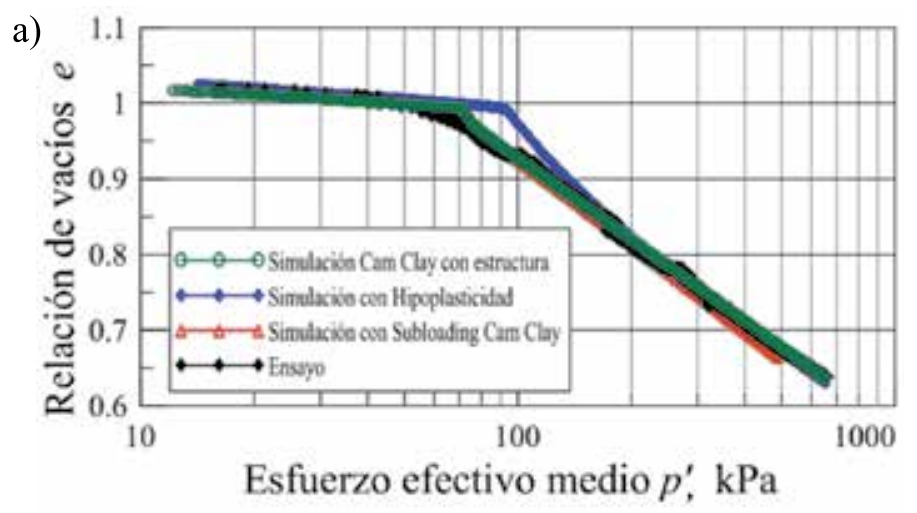

b)

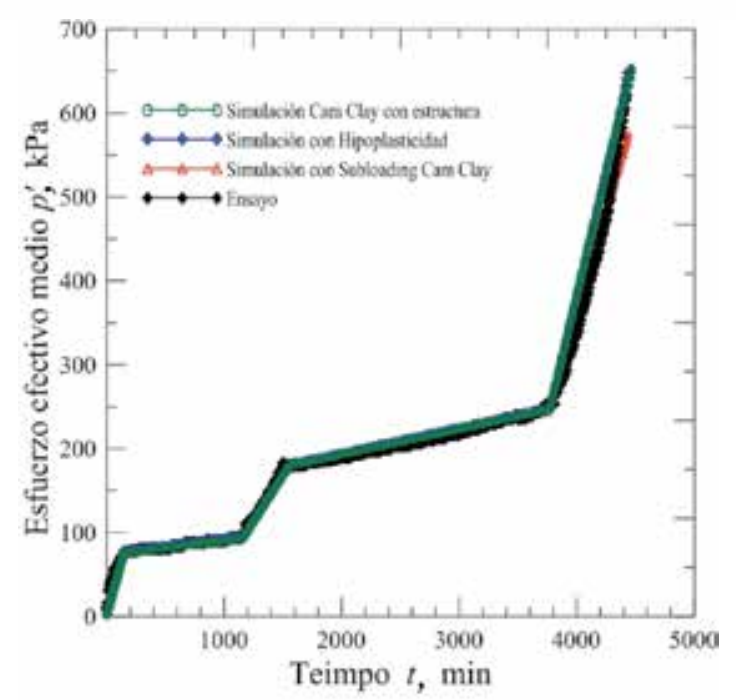

Figura 20: Comparación de simulaciones realizadas y el ensayo de consolidación con $\eta=0.5$

Por último, en la Figura 21, se realiza la comparación de las simulaciones y el ensayo, al tener un cambio en la relación de esfuerzos $(\eta=0.5$ a $\eta=0.0)$. En la Figura 21 a se observa que los modelos Cam Clay con estructura y Subloading 
captan el mismo cambio de esfuerzo de preconsolidación con el cambio de trayectoria de esfuerzos en el ensayo. Esto ocurre porque los dos modelos tienen la misma superficie de plastificación, lo que no ocurre con el modelo Hipoplástico que no tiene la misma superficie definida. Los modelos Cam Clay usados fueron los que mejor simularon el cambio del esfuerzo de preconsolidación en comparación con el ensayo. En la Figura 21b se muestra un comportamiento similar de las simulaciones en comparación con el ensayo.

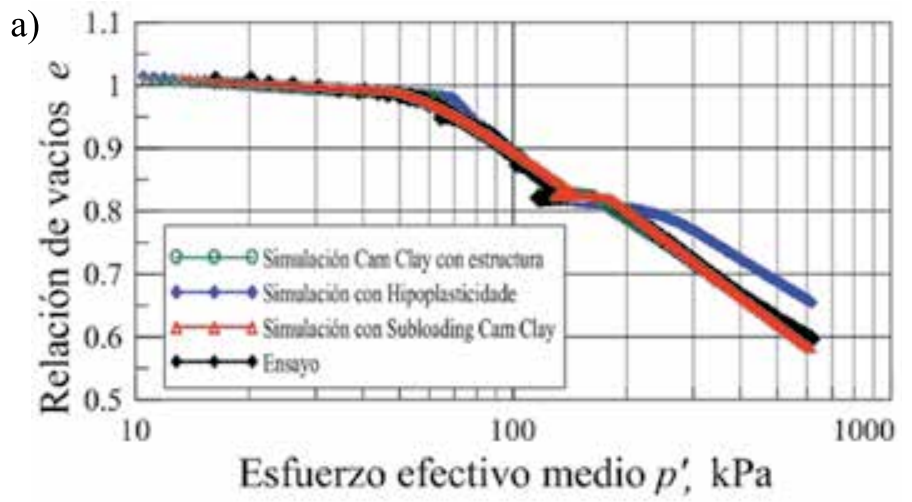

b)

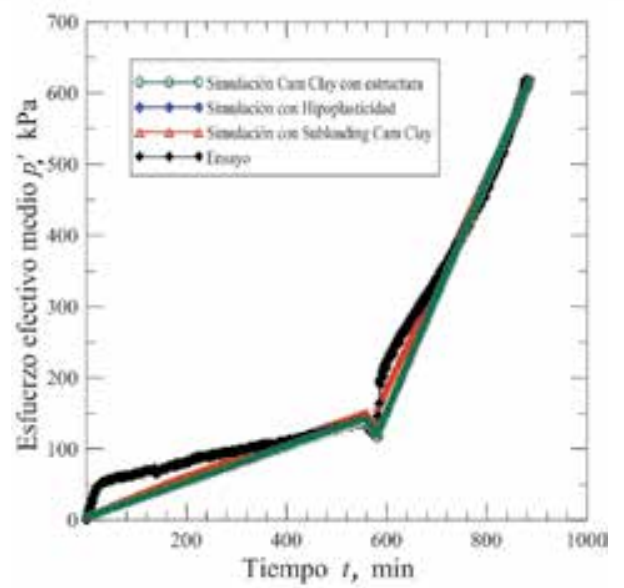

Figura 21: Comparación de simulaciones realizadas y el ensayo de consolidación con $\eta=0.5-0.0$

Se comenzó con las simulaciones de seis ensayos triaxiales con la generación de esfuerzos de corte a presiones efectivas de confinamiento de 110,200 y 300 $\mathrm{kPa}$. Los primeros tres ensayos simulados fueron bajo una condición no drenada (deformación volumétrica igual a cero), como se muestra en las Figuras 22 y 23a. Los otros tres ensayos fueron simulados con las mismas condiciones de confinamiento, pero con esfuerzos desviadores de forma drenada (Figuras 22 y 23b). En estas simulaciones se usaron los parámetros obtenidos de las simulaciones de los ensayos de consolidación (los cuales son presentados al final de la sección).

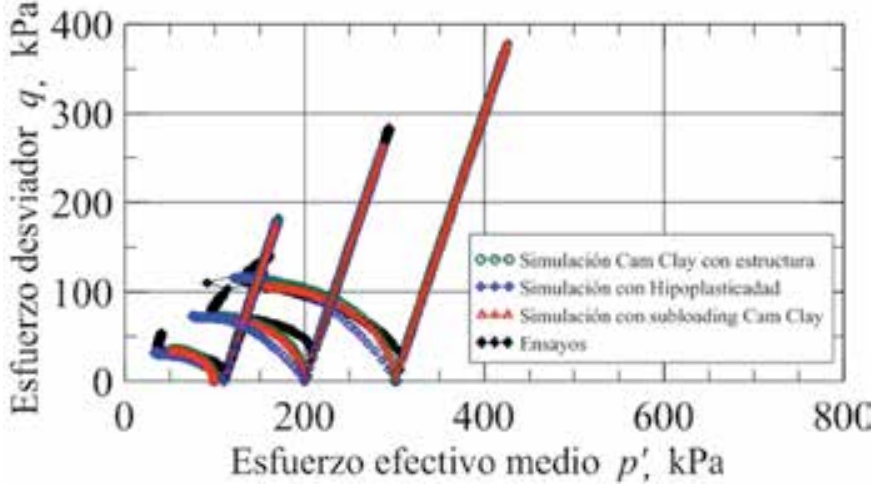

Figura 22: Trayectorias de esfuerzos simuladas de los ensayos triaxiales

a)

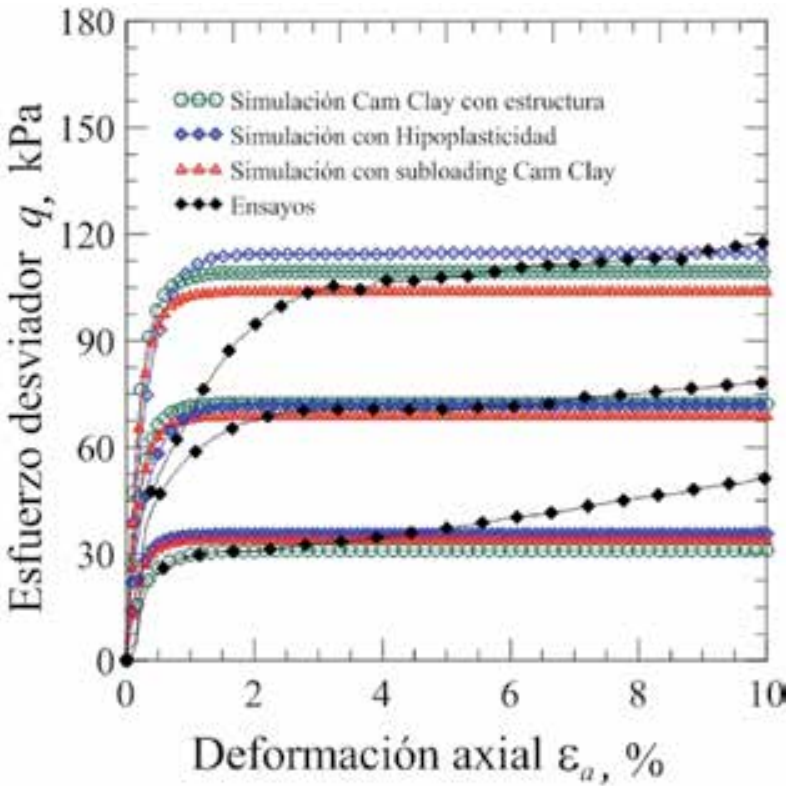

b)

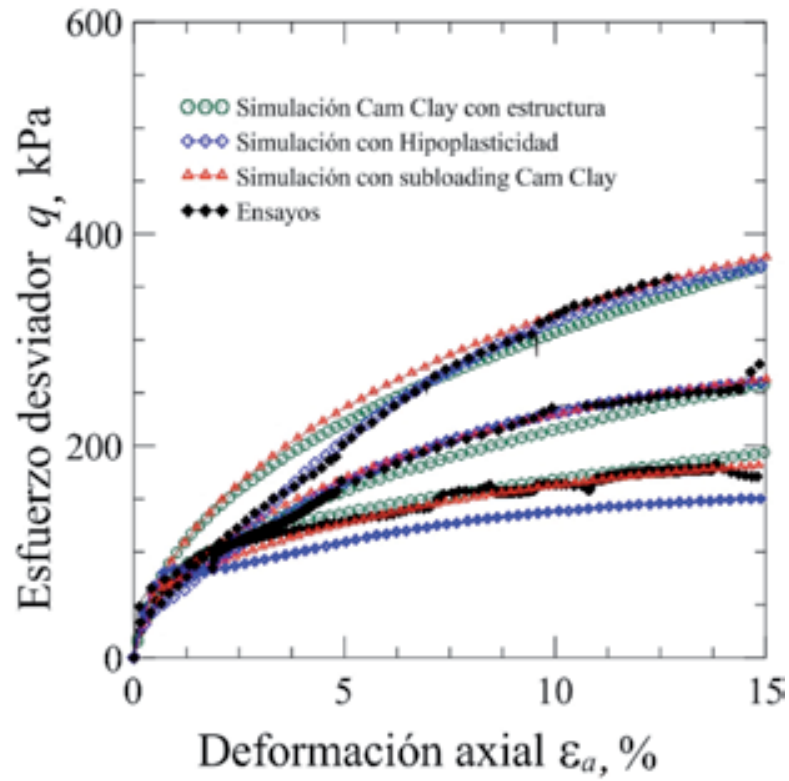

Figura 23: Comparación de simulaciones realizadas con ensayos triaxiales para: a) condiciones no drenadas y b) condiciones drenadas 
De estas comparaciones se observó que ninguno de los modelos implementados fue capaz de representar las variaciones de esfuerzo y deformación de los ensayos no drenados (Figura 23a). Sin embargo, los tres modelos presentaron variaciones de esfuerzo y deformación similares. En cuanto a los ensayos drenados se observó un desempeño mejor al comparar las variaciones de esfuerzo y deformación (Figura 23b), sin embargo el modelo hipoplástico representa un poco mejor las variaciones de deformación y esfuerzo al captar el efecto de cementación presentado en los ensayos.

\section{Análisis de resultados y discusión}

Basándose en los ensayos triaxiales realizados, se puede observar que la arcilla estructurada de la ciudad de Brasilia es poco influenciada por la variación de velocidad en los ensayos. En las Figuras 4a y 8a se encuentran las isotacas (líneas de esfuerzo-deformación con el cambio de velocidad que afectan la resistencia del suelo) al igual que en los trabajos presentados por Tatsuoka et al. (2000) y Sorensen et al. (2007). Se realizó el cálculo del porcentaje de aumento o disminución de la resistencia con el cambio de velocidad y se obtuvo un porcentaje de $3 \%$. Leinenkugel (1976) relacionó la influencia de la velocidad en la resistencia del suelo a través de la viscosidad, como es mostrado en la ecuación (45), con esta ecuación se calculó un índice de viscosidad $I_{v \alpha}=0.0135$, el cual presenta un valor bajo como se muestra en la Figura 24. La figura anterior fue obtenida del trabajo presentado por Krieg (2000) y relaciona experimentalmente el $I_{v \alpha}$ con el límite de liquidez del suelo.

$$
R_{e v}=R_{e v \alpha}\left[1+I_{v \alpha} \ln \frac{\dot{\varepsilon}}{\dot{\varepsilon}_{\alpha}}\right]
$$

De los ensayos de consolidación triaxial, se observa que el efecto de cementación está aproximadamente hasta 500 $\mathrm{kPa}$ (Figuras 3, 4 y 5) y su efecto es bajo en comparación con otros suelos reportados por Lagioia y Nova (1995) y Anagnostopoulos et al. (1991). Sin embargo, al estudiar el efecto de la cementación se obtiene un valor aproximado del $30 \%$ de influencia de la cementación en el esfuerzo de preconsolidación, lo cual puede ser importante en los diseños geotécnicos al momento de usar metodologías de comportamiento preconsolidado a normalmente consolidado e interpretar los resultados de laboratorio.
Otro aspecto importante es la influencia de la cementación en los ensayos triaxiales drenados, donde ésta hace variar los valores de deformación unitaria vertical de $1.5 \%$ hasta $7 \%$, valores típicos donde trabajan las obras geotécnicas y que afecta la rigidez del suelo.

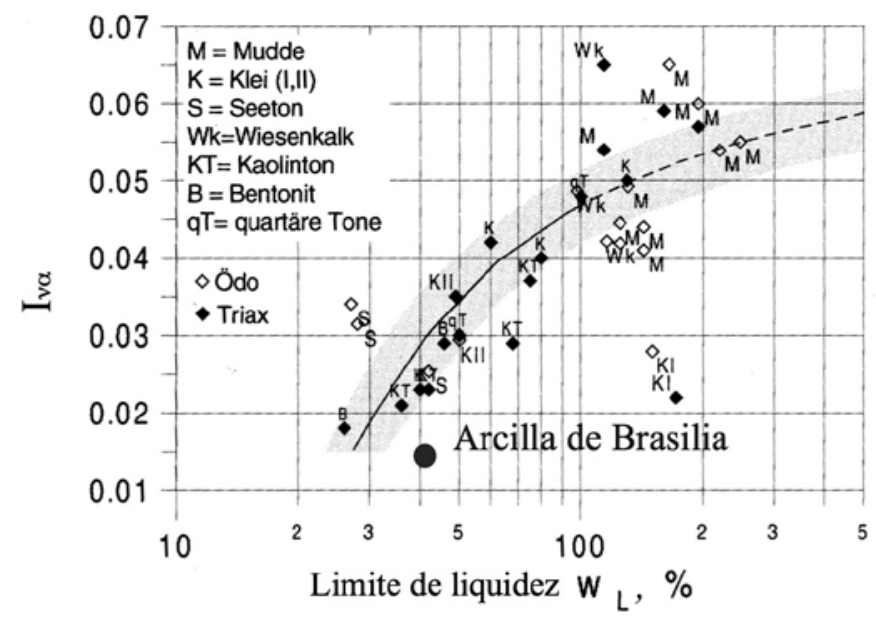

Figura 24: Límite de liquidez versus índice de viscosidad (Krieg, 2000)

El modelo Cam Clay con estructura ignora la anisotropía de esfuerzos que presenta el suelo cuando es sometido a un estado de compresión y extensión triaxial. Los modelos Subloading Cam Clay e Hipoplasticidad tienen en cuenta este tipo de anisotropía por los criterios de ruptura incorporados (criterio de Argyris-Sheng y MatsuokaNakai), al presentar una menor resistencia del suelo cuando es sometido a un esfuerzo de tensión.

El modelo Subloading Cam Clay cuenta con una regla de flujo asociada, lo cual puede ser una desventaja del modelo. Lo anterior se debe a las observaciones experimentales que evidencian una relación entre los incrementos de deformación plástica desviadora y deformación plástica volumétrica menores que los presentes en muestras reconstituidas (Liu y Carter, 2002). El modelo Cam Clay con estructura ya tiene una regla de flujo no asociada por medio de una superficie de potencial plástico diferente de la superficie de fluencia, como se mostró en la ecuación (4). El modelo Hipoplástico cuenta con una regla de flujo que está en dirección opuesta a la tasa de deformación (Masín, 2006), pero al realizar una analogía con las leyes de flujo presentadas por el modelo Cam Clay se tendría una regla de flujo no asociada (Niemunis, 2003). En los modelos de Hipoplásticidad y Subloading Cam Clay se observó un paso suave del estado elástico (estructurado) al estado 
elasto-plástico (desestructurado). En el modelo Cam Clay con estructura no ocurrió esto, al tener un cambio brusco del estado elástico al estado elasto-plástico.

\section{Conclusiones}

Basados en los ensayos triaxiales realizados y en las simulaciones se observó que los modelos estudiados pueden representar algunas características del suelo analizado, pero no todas. De las características no representadas por los modelos está el endurecimiento obtenido en los ensayos triaxiales no drenados y el relajamiento obtenido en el ensayo de consolidación. De las características obtenidas están las curvas tensión-deformación del ensayo triaxial drenado y el cambio del comportamiento preconsolidado a normalmente consolidado. Sin embargo, el modelo subloading Cam Clay presenta una transición menos rígida del cambio de éstas que se asemeja más a los resultados de los ensayos. El modelo Hipoplástico representó mejor el efecto de cementación en los ensayos triaxiales drenados y se observó una facilidad mayor al momento de realizar la implementación en tres dimensiones. Con lo anterior se crean dos nuevas opciones para seguir investigando: desarrollar otros modelos constitutivos o crear un modelo que represente mejor las características atípicas de la arcilla porosa de la ciudad de Brasilia.

\section{Agradecimientos}

Los autores agradecen al Consejo Nacional de Desarrollo Científico y Tecnológico CNPq y a la Universidad de Brasilia por el soporte técnico y financiero. El primer autor agradece a la Universidad Piloto de Colombia, al grupo de investigación $\mathrm{HD}+i$ en el proyecto de Modelación numérica de cimentaciones profundas tomando en cuenta la variabilidad de los parámetros geotécnicos por el soporte técnico y financiero.

\section{Apéndice}

La notación tensorial utilizada en este trabajo es matricial. Por definición, los tensores son transformadores lineales enmarcados dentro de un espacio (dimensiones) y se pueden representar mediante una base. Para fines de este documento se selecciona una base cartesiana con vectores unitarios $\mathrm{e}_{1}, \mathrm{e}_{2}, \mathrm{e}_{3}$. Bajo la representación tensorial, los escalares estarán denotados con letra cursiva en minúscula, p.e. $\lambda$ con excepción de algunas siglas que representan un valor escalar y se simbolizan con letra mayúscula y normal, p.e. OCR. Los tensores de segundo orden se denotan por letras en negrilla, p.e. $\sigma$ y de cuarto orden con letra Cambria Math en mayúscula, p.e. $C^{e p}$. Algunos operadores tensoriales serán introducidos a lo largo del documento. El producto punto será denotado por $\mathbf{A} \cdot \mathbf{B}=\mathbf{A}_{\mathrm{ij}} \mathbf{B}_{\mathrm{jk}}$. El doble producto punto trasponiendo un tensor, también conocido como doble contracción será definido como la traza del producto punto con el primer tensor transpuesto, $\mathbf{A}: \mathbf{B}=\operatorname{tr}\left(\mathbf{A}^{\mathrm{T}} \cdot \mathbf{B}\right)$. El símbolo $\otimes$ denota producto diádico. La traza de un tensor de segundo orden extrae la suma de los elementos con índices iguales y se representa como $\operatorname{tr} \mathrm{A}=\mathrm{A}_{\mathrm{ij}}$.

En (46) se muestra la derivada parcial de $f$ respecto a cada componente de esfuerzo y sus derivadas internas.

$$
\frac{d f}{d \boldsymbol{\sigma}}=\frac{d f}{d p} \frac{d p}{d \boldsymbol{\sigma}}+\frac{d f}{d q} \frac{d q}{d \boldsymbol{\sigma}}+\frac{d f}{d M(\theta)} \frac{d M(\theta)}{d \theta} \frac{d \theta}{d \boldsymbol{\sigma}}
$$

Derivada de $f$ respecto al esfuerzo efectivo medio $p$.

$$
\frac{d f}{d p}=M(\theta)^{2}\left(2 p-p_{1}\right)
$$

Derivada de $p$ respecto al tensor de segundo orden $\boldsymbol{\sigma}$.

$$
\frac{d p}{d \boldsymbol{\sigma}}=\frac{1}{3} \mathbf{1}
$$

Derivada de $f$ respecto al esfuerzo desviador $q$.

$$
\frac{d f}{d q}=2 q
$$

Derivada de $q$ respecto al tensor de esfuerzos $\boldsymbol{\sigma}$.

$$
\frac{d q}{d \boldsymbol{\sigma}}=\sqrt{\frac{3}{2}} \frac{\xi}{\|\xi\|}
$$

Derivada de $f$ respecto a la pendiente del estado crítico afectado por el ángulo de Lode $M(\theta)$.

$$
\frac{d f}{d M(\theta)}=2 M(\theta) p\left(p-p_{1}\right)
$$

Derivada de $M(\theta)$ respecto al ángulo de Lode $\theta$.

$$
\frac{d M(\theta)}{d \theta}=\frac{0.75 M(1-w) \cos 3 \theta}{1+w-(1-w) \sin 3 \theta}
$$

Derivada de $\theta$ respecto al tensor de segundo orden $\boldsymbol{\sigma}$. 


$$
\frac{d \theta}{d \sigma}=\frac{1.5}{q^{2} \cos 3 \theta}\left(\frac{3}{q} \operatorname{dev} \xi^{2}-\sin 3 \theta \xi\right)
$$

Se dan las derivadas respecto a la función de potencial plástico g para implementar el modelo CCS.

$$
\begin{gathered}
\frac{d g}{d \boldsymbol{\sigma}}=\frac{d g}{d p} \frac{d p}{d \boldsymbol{\sigma}}+\frac{d g}{d q} \frac{d q}{d \boldsymbol{\sigma}} \\
\frac{d g}{d p}=2 M(\theta)^{2} p-M(\theta)^{2} p_{y, i}^{\frac{1}{a}}\left(\frac{2 a-1}{a}\right) p^{\frac{a-1}{a}} \\
a=\frac{(1-\omega \Delta e)}{(1-2 \omega \Delta e)} \\
\frac{d g}{d q}=2(1-2 \omega \Delta e) q
\end{gathered}
$$

\section{Referencias}

Anagnostopoulos, A.G., Kalteziotis, N., Tsiambaos, G.K. and Kavvadas, M. (1991). Geotechnical properties of the Corinth canal marls. Geotechnical and Geological Engineering 9(1), $1-26$

Baudet, B.A. and Stallebrass, S.E. (2004). A constitutive model for structured clays. Géotechnique 54(4), 269-278

Burland, J.B. (1990). On the compressibility and shear strength of natural clays. Géotechnique 40(3), 329-378

Camapum de Carvalho, J., Martines M., Moreira de Souza, N. e da Silva M. (2006). Processos Erosivos no Centro-Oeste Brasileiro. Universidade de Brasília (en portugués)

Cuccovillo, T. and Coop, M.R. (1999). On the mechanics of structured sands. Géotechnique 49(6), 741-760

Farias, M.M., Pedroso, D.M. and Nakai, T. (2009). Automatic substepping integration of the subloading tij model with stress path dependent hardening. Computers and Geotechnics 36, 537-548

Fuentes, W., Mendoza, C. and Lizcano, A. (2010). Evaluation of an extended viscohypoplastic model for structured soils. $X V$ Congresso Brasileiro de Mecânica dos Solos e Engenharia Geotécnica, [DC-Room], Brasília, Brasil

Giraldo, R. e Farias, M. (2011). Validação experimental de um modelo simples para solos. VI INFOGEO - Simpósio Brasileiro de Aplicações de Informatica em Geotecnia, 1, 1
Guimarães, R. (2002). Análise das propriedades e comportamento de um perfil de solo laterítico aplicada ao estudo do desempenho de estacas escavadas. Tesis MSc, Universidade de Brasília (en portugués)

Helwany, S. (2007). Applied Soil Mechanics with ABAQUS Applications. 1st edition. John Wiley and Sons, Inc.

Kolymbas, D. (1977). Ein nichtlineares viskoplastisches Stoffgesetz für Boden. $\mathrm{PhD}$ thesis, Insitut für Boden und Felsmechanik, University of Karlsruhe, Germany (en alemán)

Krieg, S. (2000). Viskoses Bodenverhalten von Mudden, Seeton und Klei. PhD thesis, University of Karlsruhe, Germany (en alemán)

Lagioia, R. and Nova, R. (1995). An experimental and theoretical study of the behaviour of a calcarenite in triaxial compression. Géotechnique 45(4), 633-648

Leinenkugel, H.J. (1976). Deformation and strength behavior of cohesive soils experiments and their physical meaning. Tech. Rep. Heft 66, Institute of Soil and Rock Mechanics, University of Karlsruhe, Germany

Leroueil, S. and Vaughan, P.R. (1990). The general and congruent effects of structure in natural soils and weak rocks. Géotechnique 40(3), 467-488

Liu, M.D. and Carter, J.P. (2002). A structured Cam Clay model. Canadian Geotechnical Journal 39(6), 1313-1332

Liu, M.D. and Carter, J.P. (2006). A structured Cam Clay model. Research report $N^{\circ}$ R814, University of Sydney, Australia.

Liyanapathirana, S., Carter, J. and Airey, D. (2005). Numerical modeling of nonhomogeneous behaviour of structured soils in triaxial tests. International Journal of Geomechanics 5(1), 10-23 Masín, D. (2006). Hypoplastic models for fine-grained soils. $\mathrm{PhD}$ thesis, Charles University, Prague, Czech Republic

Melfi, A.J. (1997). Lateritas e Processos de Laterização. Escola de Engenharia de São Carlos, Universidade de São Paulo (en portugués)

Mendoza, C.C. (2013). Experimental and numerical behaviour of deep foundations made up by "Alluvial Anker" type piles founded in a tropical soil of Brazil. $\mathrm{PhD}$ thesis, University of Brasília (in Portuguese)

Nakai, T. and Hinokio, M. (2004). A simple elastoplastic model for normally and over consolidated soils with unified material parameters. Soils and Foundations 44(2): 12-30 
Nakai, T., Shahin, H.M., Kikumoto, M., Kyokawa, H. and Zhang, F. (2009). Simple and unified method for describing various characteristics of geomaterials - Influences of density, bonding, time effects and others. Journal of Applied Mechanics JSCE 12, 371- 382 (in Japanese)

Niemunis, A. (2008). Incremental Driver, user's manual. Karlsruhe Institute of Technology KIT

Niemunis, A. (2003). Extended hypoplastic models for soils. Institut für Grundbau und Bodenmechanik, Ruhr Universitat Bochum, Germany

Pedroso, D.M. (2006). Representação matemática do comportamento mecánico cíclico de solos saturados e não saturados. Tesis $\mathrm{PhD}$, Universidade de Brasília (en portugués)

Roscoe, K.H., Schofield, A.N. and Wroth, C.P. (1958). On the yielding of soils. Géotechnique 8(1), 22-52

Roscoe, K.H., Schofield, A.N. and Thurairajah, A. (1963). Yielding of clays in states wetter than critical. Géotechnique 13(3), 211-240

Roscoe, K.H., and Burland, J.B. (1968). On the generalized stress-strain behavior of "wet clay". In Engineering Plasticity. Edited by J. Herman and F.A. Leckie. Cambridge University Press, 535-609

Sheng, D., Sloan S.W. and Yu, H.S. (2000). Aspects of finite element implementation of critical state models. Computational Mechanics 26(2), 185-196
Sorensen, K.K., Baudet, S. and Simpson, B. (2007). Influence of structure on the time-dependent behaviour of a stiff sedimentary clay. Géotechnique 57(1), 113-124

Tatsuoka, F., Santucci de Magistris, F., Hayano, K., Momoya, Y. and Koseki., J. (2000). Some new aspects of time effects on the stress and strain behavior of stiff geomaterials. Proceedings of the Second International Conference on Hard Soils - Soft Rocks, Napoli, Vol. 2, 1285-1371

Vatsala, A., Nova, R. and Murthy, B.R.S. (2001). Elastoplastic model for cemented soils. Journal of Geotechnical and Geoenvironmental Engineering 127(8), 679-687

Wolffersdorff, P.A. (1996), A hypoplastic equation for granular materials with a predefined limit state surface. Mechanics of Cohesive-Frictional Materials 1(3), 251-271

Whitlow, R. (2000). Basic soil mechanics. Prentice Hall, 4th ed. Wu, W. (1992). Hypoplastizität als mathematisches Modell zum mechanischen Verhalten granularer Stoffe. $\mathrm{PhD}$ thesis, Insitut für Boden und Felsmechanik, University of Karlsruhe, Germany (en alemán)

Yan, W.M. and Li, X.S. (2011). A model for natural soil with bonds. Géotechnique 61(2), 95-106 